\title{
Electrophysiological Imaging of Functional Architecture in the Cortical Middle Temporal Visual Area of Cebus apella Monkey
}

\author{
Antonia Cinira M. Diogo, ${ }^{1,3}$ Juliana G. M. Soares, ${ }^{1}$ Alex Koulakov, ${ }^{2}$ Thomas D. Albright, ${ }^{3}$ and Ricardo Gattass ${ }^{1}$ \\ ${ }^{1}$ Instituto de Biofísica Carlos Chagas Filho, Universidade Federal do Rio de Janeiro, Rio de Janeiro 21941-900, Brazil, and ${ }^{2}$ Sloan-Swartz Center for \\ Theoretical Neurobiology and ${ }^{3}$ Howard Hughes Medical Institute and Systems Neurobiology Laboratories, The Salk Institute for Biological Studies, La Jolla, \\ California 92037
}

\begin{abstract}
We studied the spatial organization of directionally selective neurons in the cortical middle temporal visual area (area MT) of the Cebus monkey. We recorded neuronal activity from multielectrode arrays as they were stepped through area MT. The set of recording sites in each array penetration described a plane parallel to the cortical layers. At each recording site, we determined the preferred direction of motion. Responses recorded at successive locations from the same electrode in the array revealed gradual changes in preferred direction, along with occasional directional reversals. Comparisons of responses from adjacent electrodes at successive locations enabled electrophysiological imaging of the two-dimensional pattern of preferred directions across the cortex. Our results demonstrate a systematic organization for directionality in area MT of the New World Cebus monkey, which is similar to that known to exist in the Old World macaque. In addition, our results provide electrophysiological confirmation of map features that have been documented in other cortical areas and primate species by optical imaging. Specifically, the tangential organization of directional selectivity is characterized by slow continuous changes in directional preference, as well as lines (fractures) and points (singularities) that fragment continuous regions into patches. These electrophysiological methods also allowed a direct investigation of neuronal selectivities that give rise to map features. In particular, our results suggest that inhibitory mechanisms may be involved in the generation of fractures and singularities.
\end{abstract}

Key words: extrastriate cortex; directional selectivity; visual system; primates; multielectrode array; functional maps

\section{Introduction}

A fundamental feature of neocortical organization is the arrangement of neurons representing similar attributes into columns (Mountcastle, 1957; Hubel and Wiesel, 1962, 1974a). These columns have been investigated extensively by electrophysiological recording of neuronal responses along individual microelectrode penetrations. Because each electrode provides a one-dimensional (1D) view, however, it has been difficult to deduce the form of the larger two-dimensional (2D) representation (Hubel and Wiesel, 1974a; Braitenberg and Braitenberg, 1979). Optical imaging (OI) offers an alternative means to assess 2D architecture. For example, optical images of orientation selectivity in primary visual cortex (V1) have revealed a characteristic radial structure, and evidence for fractures (abrupt changes in preferred orientation) and singularities (points of convergence of several different iso-orientation columns) (Blasdel and Salama,

\footnotetext{
Received Aug. 9, 2002; revised Feb. 21, 2003; accepted Feb. 24, 2003.

This work was supported by the Brazilian government through the Fundação Carlos Chagas Filho de Amparo à Pesquisa do Estado do Rio de Janiero, Conselho de Ensino para Graduados e Pesquina da Universidade Federal do Rio de Janiero, Conselho Nacional de Desenvolvimento Cientifico e Tecnológico, Financiadora de Estudos e Projetos, and Programa de Apoio a Núcleos de Excelêcia, and by the National Eye Institute of the National Institutes of Health. T.D.A. is an Investigator of the Howard Hughes Medical Institute. We are grateful to Mario Fiorani, Jay Hegde, Greg Horwitz, Bart Krekelberg, Ralph Siegel, and Jean Christophe Houzouel for valuable comments on this manuscript. We also thank Edil Saturato da Silva Filho, Liliane Heringer Pontes, and Maria Thereza Alves Monteiro for skillfu technical assistance, and Mario Fiorani Jr and Giuseppe Bertini for sharing their Matlab routines for data analysis.

Correspondence should be addressed to Dr. Ricardo Gattass, Instituto de Biofísica Carlos Chagas Filho, Universidade Federal do Rio de Janeiro, Rio de Janeiro, 21941-900, Brazil. E-mail: rgattass@biof.ufrj.br.

Copyright $\odot 2003$ Society for Neuroscience $\quad$ 0270-6474/03/233881-18 $\$ 15.00 / 0$
}

1986; Ts'o et al., 1990; Bonhoeffer and Grinvald, 1991; Malonek et al., 1994; Weliky et al., 1996).

There are, however, limitations to the optical approach. One obvious problem is that optical signals cannot be recorded from cortical regions that are buried within sulci. Some investigators have thus turned to the lissencephalic New World owl monkey (Aotus), but this nocturnal primate is less suitable than others as a model of human brain organization and function. A second problem with the optical approach is that individual neuronal response properties are not accessible directly.

These limitations motivated us to develop an electrophysiological approach that is complementary to optical imaging. Our approach involves the use of multiple microelectrodes arranged in compact arrays and moved simultaneously parallel to the cortical laminas. This method yields a 2D sample of neuronal selectivity - sequentially along each electrode and simultaneously across all electrodes of the array (see Fig. 1)-within the cortical plane. These electrophysiological measurements can be used to interpolate continuous functional maps similar to those obtained from optical imaging. Not only does this technique offer a means to investigate optically inaccessible regions of cortex, it may also reveal the specific neuronal selectivity patterns that give rise to map features.

We have used electrophysiological imaging (EI) to study the 2D representation of visual motion in area MT of Cebus apella, a diurnal New World monkey with a gyrencephalic brain that is morphologically similar to that of the Old World macaque. Albright et al. (1984) demonstrated that neurons in area MT of 
Macaca fascicularis are organized into columns of similar preferred direction and axis of motion. Although the 1D electrophysiological data obtained by Albright et al. (1984) were insufficient to reconstruct $2 \mathrm{D}$ functional maps for area MT, these investigators hypothesized a rectilinear columnar arrangement, similar to the original ice-cube model proposed for V1 (Hubel and Wiesel, 1974a). Unfortunately, hypotheses regarding functional maps in area MT cannot be evaluated by optical imaging in primates such as Cebus and Macaca, in which this area is buried in a sulcus.

Electrophysiological imaging has allowed us to advance beyond these findings in three important ways. First, it has afforded an unprecedented opportunity to assess 2D functional maps in a primate in which area MT is optically inaccessible. Second, our electrophysiological approach has allowed us to identify characteristics of neuronal selectivity that give rise to the 2D maps. Third, our discovery of functional maps in the New World Cebus monkey has enabled us to document their resemblance to those of the Old World macaque, which suggests a common evolutionary adaptation.

\section{Materials and Methods \\ Animal subjects}

We recorded neuronal activity from five adult Cebus monkeys (Cebus apella), each weighing between 2 and $3 \mathrm{~kg}$. Each animal was a subject in three to five recording sessions. All experimental protocols followed $\mathrm{Na}$ tional Institutes of Health guidelines for animal care and use and were approved by the Institutional Animal Care and Use Committee at Instituto de Biofísica Carlos Chagas Filho/Universidade Federal do Rio de Janeiro.

\section{Animal preparation and maintenance}

General procedures were similar to those used by Albright et al. (1984). Briefly, 1 week before the first recording session, a stainless-steel cylinder and a head bolt, oriented in the stereotaxic planes, were affixed to the animal's skull with screws and dental acrylic. Surgical procedures were performed under aseptic conditions using ketamine anesthesia $(20 \mathrm{mg} /$ $\mathrm{kg}$ ). For the recording sessions, animals were anesthetized initially using ketamine $(20 \mathrm{mg} / \mathrm{kg})$ followed by halothane $(2.0 \%)$ in a $7: 3$ mixture of nitrous oxide and oxygen. Animals were paralyzed by a continuous infusion of pancuronium bromide $\left(0.1 \mathrm{mg} \cdot \mathrm{kg}^{-1} \cdot \mathrm{hr}^{-1}\right)$ and artificially ventilated. Halothane was discontinued once paralysis became stable, and anesthesia was maintained by nitrous oxide and oxygen and by continuous infusion of fentanyl citrate $\left(0.003 \mathrm{mg} \cdot \mathrm{kg}^{-1} \cdot \mathrm{hr}^{-1}\right)$. Body temperature was maintained at $37-38^{\circ} \mathrm{C}$ with a heating pad, and respiratory parameters were adjusted to give an end-tidal carbon dioxide level of $4 \%$. The head of the monkey was held firmly in a stereotaxic apparatus by means of the head bolt. Before visual stimulation began, the cornea was fitted with a contact lens and accommodation was paralyzed by topical application of atropine (1\%). The contralateral eye was focused on a tangent screen at a distance of $57 \mathrm{~cm}$, and the ipsilateral eye was occluded.

Recording sessions generally continued for 10-18 hr. One hour before the end of the experiment, the paralytic agent was discontinued. The recording cylinder was washed out and filled with saline, and the animal was allowed to recover. Usually within $3 \mathrm{hr}$, the animal was alert and active in its home cage. Successive recording sessions were separated by at least 1 week.

\section{Microelectrode arrays for electrophysiological imaging}

We used 1D microelectrode arrays to sample neuronal response properties at nodes within a $2 \mathrm{D}$ rectilinear matrix (Fig. 1). The arrays were constructed by gluing together a set of microelectrode guide tubes ( 6 or 11 guide tubes in two different configurations, as described below and shown in Fig. $1 \mathrm{~A}$ ), such that the electrode tips formed points on a line or nearly so (estimated error, $\pm 30 \mu \mathrm{m}$ ). Each array was advanced parallel to the cortical surface in $200 \mu \mathrm{m}$ steps, and neuronal responses were recorded from each electrode following each step of the array. Although each row of the matrix was thus sampled at a different point in time-

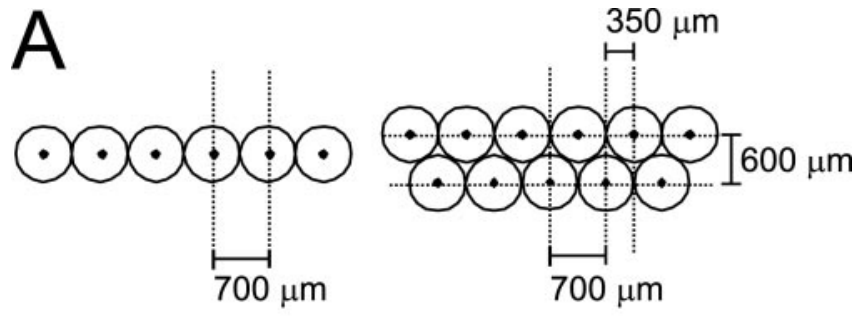

E I

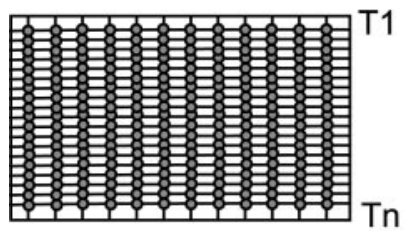

$200 \mu \mathrm{m} \times 350 \mu \mathrm{m}$ $1 \mathrm{~ms}$

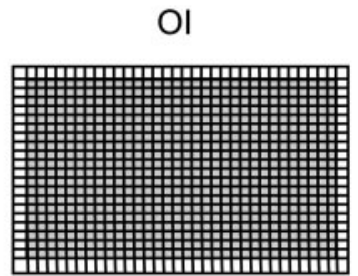

$150 \mu \mathrm{m} \times 150 \mu \mathrm{m}$ $500 \mathrm{~ms}$
Figure 1. Schematic comparison of El and $01 . A$, Multielectrode arrays used for El. The array shown at left contains six electrodes spaced $700 \mu \mathrm{m}$ apart. The array shown at right contains 11 electrodes that lie in two parallel interdigitating planes, which permits recordings spaced at 350 $\mu \mathrm{m}$ relative to cortical laminas. $B$, Left, Electrophysiological imaging. Electrodes are moved simultaneously through a region of cortex, stopping at predetermined positions (e.g., every 200 $\mu \mathrm{m}$ ). At each position, neuronal responses to a specific set of stimulus conditions (e.g., different directions of stimulus motion) are recorded. The recording sites at each point in time (small gray circles) thus describe a line (1 row). After the electrode array has crossed the cortical region, the set of recording sites describes a plane, rows of which have been sampled at sequential points in time. The sampling resolution of the matrix of neuronal tuning data is determined by the distance between electrodes in the array (columns in this figure) and the distance between positions of the array (rows in this figure) as it is advanced through the cortex. Spatial and temporal sampling resolutions for the data obtained from the present application of El are indicated below the El array. B, Right, Optical imaging. This more conventional technique obtains the entire tuning matrix at a single point in time. Spatial and temporal sampling resolutions for typical 0 applications are indicated below the 0 l array. As implemented, the two methods also differ in spatial resolution (Ol is greater), temporal resolution (El is greater), and signal source (electrophysiological, extracellular action potentials; vs optical, membrane potential). T1, First recording in a given multiple electrode penetration; Tn, last recording.

unlike optical imaging in which all samples in the 2D matrix are obtained simultaneously - the final product of this procedure was a 2D matrix of samples (Fig. $1 B$, left). The spatial resolution of this matrix was either $200 \times 700$ or $200 \times 350 \mu \mathrm{m}$, depending on the electrode array configuration (see below and Fig. $1 A$ ).

In contrast to the electrophysiological imaging applied in these experiments, optical imaging has a somewhat greater spatial resolution (Fig. $1 B$, right), commonly in the range of $150 \times 150 \mu \mathrm{m}$ (Ts'o et al., 1990) but potentially as fine as the distance between blood vessels (i.e., up to $50 \times$ $50 \mu \mathrm{m}$ ) (Grinvald et al., 2001). The temporal resolution of extracellular electrophysiological signals is, however, far superior to that of optically recorded intrinsic signals (Ts'o et al., 1990; Shmuel and Grinvald, 1996).

\section{Electrophysiological recording strategies}

We used two different arrays and multiunit recording strategies. In three animals (monkeys A, B, and C), the array contained six microelectrodes that were spaced $700 \mu \mathrm{m}$ apart (Fig. $1 A$, left). Neuronal response properties were assessed qualitatively: visual stimuli were presented manually using a handheld projector and rear-projection screen. Patterns of stimulus selectivity seen on each electrode were characterized by the experimenter based on an audio evaluation of firing rate under different conditions. After the neuronal receptive field (RF) boundaries were mapped, the preferred direction was determined by judging the best multiunit response to a bar that was moved in systematically varied directions. The angle of the preferred direction was measured with the aid of an extended protractor. If the site exhibited no strong responses compared with spontaneous activity in all tested directions, it was classified as nonresponsive. 
If the site was responsive to moving stimuli but manifested no clear directional preference, it was classified as pandirectional. Some recording sites had two preferred directions that were clearly better than others. We classified these sites as bidirectional. In such cases, we determined the preferred direction to be the one eliciting stronger responses.

Recordings in monkeys $\mathrm{A}, \mathrm{B}$, and $\mathrm{C}$ were primarily confined to the cortex representing an area of the visual field corresponding to the lower quadrant contralateral to the recorded hemisphere and within $\sim 10^{\circ}$ of the fovea. Microelectrode array penetrations entered the brain from the dorsocaudal direction at an angle of $28^{\circ}$ posterior with respect to the frontal plane. One penetration in monkey $\mathrm{B}$ was made in the same $28^{\circ}$ oblique-frontal plane but entered the brain from a direction $30^{\circ}$ lateral to the sagittal plane. All penetrations in these three animals were approximately parallel (within $5-10^{\circ}$ ) to the laminar boundaries of area MT.

In two additional animals (monkeys $\mathrm{D}$ and $\mathrm{E}$ ), the array contained 11 microelectrodes that were placed in two parallel interdigitating planes, such that their tips were staggered (Fig. $1 A$, right). Tip spacing within each plane was $700 \mu \mathrm{m}$, and the planes were separated by $600 \mu \mathrm{m}$ oncenter. Collapsing across planes, the electrode tip spacing was $350 \mu \mathrm{m}$. Thus, if the two planes of electrodes were aligned (as intended) parallel to the cortical surface, the electrode tip spacing relative to that surface was $350 \mu \mathrm{m}$. For the two animals studied using this type of array, RFs were initially characterized by hand mapping. Patterns of stimulus selectivity were subsequently assessed quantitatively using computer-controlled stimulus presentation and data acquisition (see below). Recordings in monkeys D and E were primarily confined to the cortex representing an area of the visual field corresponding to the lower quadrant contralateral to the recorded hemisphere and within $\sim 15^{\circ}$ of the fovea. In monkey $D$, the microelectrode array entered the brain from the dorsocaudal direction at an angle of $20^{\circ}$ posterior with respect to the frontal plane. In monkey E, the corresponding penetration angle was $12^{\circ}$. In both monkeys, the penetrations were approximately parallel (within $10-12^{\circ}$ ) to the laminar boundaries of area MT.

\section{Quantitative measurements of stimulus selectivity}

For monkeys D and E, patterns of selectivity were characterized using visual stimuli presented on a video monitor under computer control. Multiunit action potentials were digitized and stored in a computer. Directional selectivity and other response parameters were extracted afterward using standard parametric analyses.

Computer-generated visual stimulation. The computer-generated stimuli used for monkeys D and $\mathrm{E}$ were of two types: asymmetric square-wave gratings $\left(1.1 \mathrm{cycle} /{ }^{\circ}\right.$; duty cycle $\left.=0.33\right)$ and random-dot arrays $(\operatorname{dot}$ size $=0.5^{\circ} ; \operatorname{dot}$ density $\left.=0.45 \mathrm{dot} / \mathrm{deg}^{2}\right)$. Stimuli were moved at $3 \%$ sec in systematically varied directions ( 12 directions; $30^{\circ}$ apart) and were presented on a 21 inch raster-scan video monitor (frame rate $=60 \mathrm{~Hz}$ ) that was positioned $57 \mathrm{~cm}$ from the nodal point of the animal's eye. Stimuli were viewed through a circular aperture $25^{\circ}$ in diameter and encompassed simultaneously the classical RFs of all units recorded at each position of the multiple electrode array. Each stimulus (type and direction of motion) appeared for 10 trials on a pseudorandom schedule. Each trial contained three epochs: the video display was initially blank for 200 $\mathrm{msec}$, the stimulus then appeared without moving for $400 \mathrm{msec}$, and finally, the stimulus moved continuously in one direction for an additional $1000 \mathrm{msec}$. Neuronal responses to gratings and dots were recorded in separate blocks of trials.

Statistical analysis. Neuronal responses in monkeys D and E to moving stimuli were computed as the mean firing rate observed over the duration of movement. Spontaneous neuronal activity was computed as the mean firing rate (across all conditions) within the trial epoch in which no stimulus appeared on the video display. A paired $t$ test was used to determine whether the response in each tested direction was different from the spontaneous activity. Recording sites for which this test was nonsignificant $(p>0.05)$ in all tested directions were deemed unresponsive. Responsive recording sites were also tested using ANOVA to determine whether the response to any one direction was statistically different from that to the others. If no response difference exceeded criterion ( $p=$ $0.05)$, the recording site was classified as pandirectional. If two opposing directions elicited responses that were significantly greater than all others, the recording site was classified as bidirectional.

Various population measures of neuronal activity and selectivity were also compared under different experimental conditions. Unless indicated otherwise, the means of these population measures were compared statistically using a paired $t$ test.

Quantitative analyses of direction tuning. We began quantitative analyses of directional selectivity in monkeys $\mathrm{D}$ and $\mathrm{E}$ by fitting parametric curves to neuronal responses as a function of the direction of stimulus motion. These fits were made using a Gaussian function of the following type:

$$
r_{\mathrm{i}}=a+b e^{-0.5\left(\frac{x_{\mathrm{i}}-x_{0}}{s}\right)^{2}}
$$

where $a$ represents the minimum firing rate, $b$ represents the difference between the maximum and minimum firing rate, $x_{\mathrm{o}}$ represents the preferred direction of motion, $s$ represents the SD of the fitted Gaussian, and $r_{\mathrm{i}}$ represents the firing rate for a stimulus moving in a given direction $x_{\mathrm{i}}$. The Gaussian function that achieved the best fit to the neuronal responses in the 12 tested directions was determined for each tuning curve using an iterative least-squared-residuals algorithm. Parameters of the fitted Gaussian were used to compute measures that characterize directional tuning: differential response, bandwidth, and directional index. Differential response was the difference between the fitted maximum and minimum responses (parameter $b$ of the fitted Gaussian). [In practice, we found the minimum of the fitted Gaussian (parameter $a$ ) to be a good estimate of the minimum response recorded.] Bandwidth was the full width of the tuning curve at one-half of the distance between the maximum and minimum responses (i.e., $2.355 \times s$, where $s$ is a parameter of the fitted Gaussian). The directionality index (DI) reflects the ratio of response strength in the preferred direction relative to that in the opposite direction $\left(180^{\circ}\right.$ from preferred). This index was calculated by the following equation: DI $=1-$ (opposite direction response/preferred direction response).

\section{Histology}

At the end of a 3-5 week recording period, each animal was anesthetized with an overdose of sodium pentobarbital and perfused through the heart with saline followed by formalin solution. The brain was removed from the skull and sectioned at an angle of either $28^{\circ}$ (monkeys A, B, and C) or $20^{\circ}$ (monkeys D and E) posterior to the frontal plane. Sections were cut at $40 \mu \mathrm{m}$ thickness. Alternate sections were stained for myelin by the Gallyas (1979) method or by using a Nissl stain for cell bodies. The boundaries of area MT were determined on the basis of characteristic myeloarchitecture (Ungerleider and Mishkin, 1979; Gattass and Gross, 1981; Van Essen et al., 1981; Fiorani et al., 1989). Microelectrode tracks were reconstructed from the positions of electrolytic lesions, which were made at known points along each penetration (generally at the end), and using stereotaxic coordinates. Data analysis was restricted to recording sites located in area MT, as defined by the heavily myelinated region along the floor and lower bank of the superior temporal sulcus.

\section{Constructing functional maps of motion selectivity}

The 2D functional organization of motion selectivity in area MT was revealed by maps derived separately from the two sets of recordings [qualitative RF measures (monkeys $\mathrm{A}, \mathrm{B}$, and $\mathrm{C}$ ) and quantitative RF measures (monkeys D and E)]. In some cases, maps were constructed for both preferred direction of motion and preferred axis of motion. Preferred direction was assessed (qualitatively or quantitatively) as described above. For unidirectional recording sites, preferred axis of motion was calculated directly from the preferred direction: if the preferred direction was $\leq 180^{\circ}$, axial preference equaled directional preference; otherwise, axial preference equaled directional preference minus $180^{\circ}$. For bidirectional recording sites, preferred axis of motion was defined as the smaller (i.e., $<180^{\circ}$ ) of the two preferred directions.

Functional maps derived from qualitative RF measurements. Data from monkeys $\mathrm{A}, \mathrm{B}$, and $\mathrm{C}$ were obtained using the six-electrode array and consisted of hand-mapped RF and directional preference measurements. Using information derived from the reconstructed microelectrode penetrations, measurements of preferred direction were projected onto rep- 
resentations of the 2D cortical surface of area MT. Preferred direction of motion at each recording site was represented by an oriented arrow icon; preferred axis of movement was represented by an oriented bar icon. Asterisks were used to represent pandirectional recording sites. Nonresponsive recording sites were excluded. Because the spatial resolution of sampling was relatively low $(200 \times 700 \mu \mathrm{m})$ and precise neuronal firing rates were unavailable, we did not attempt to interpolate continuous $2 \mathrm{D}$ functional maps from this data set.

We examined the qualitative maps for the presence of architectural features (e.g., 1D sequence regularities, 2D pinwheels, and fractures) that have been identified previously in other visual areas through optical imaging. Smooth 1D sequences were readily detectable by visual inspection of direction and axis-of-motion icons along each electrode penetration in the 2D map. Two-dimensional pinwheel formations naturally required that patterns be pieced together across adjacent electrodes. For this purpose, we used visual inspection for the identification of possible radial symmetries of direction or axis-of-motion icons.

Functional maps derived from quantitative RF measurements. Data from monkeys $\mathrm{D}$ and $\mathrm{E}$ were obtained using the 11 electrode array and consisted of a matrix of computer-mapped RF measurements and directional tuning curves. This matrix typically contained $11 \times 12$ sampling nodes ( 11 electrodes $\times 12$ samples at $200 \mu \mathrm{m}$ intervals). Using information derived from the reconstructed microelectrode penetrations, measurements of preferred direction at each node in the sampling matrix were projected onto representations of the $2 \mathrm{D}$ cortical surface of area MT. Between these discrete nodes, we interpolated responses to visual motion and then used the interpolated responses to construct $2 \mathrm{D}$ maps of preferred direction.

Interpolation of neuronal responses to visual motion. To produce singlecondition (i.e., single-direction) maps of neuronal responses, we first normalized responses to the 12 tested directions at each recording site, relative to the largest of the 12 responses at the same site. This normalization was performed separately for dot and grating stimuli. This local normalization preserved information about directionality for each site, while preventing the emergence of patterns in the $2 \mathrm{D}$ activity map that merely reflect response variability from site to site.

Next, we constructed a set of 12 2D maps of normalized neuronal activity, one for each of the 12 tested directions of motion, by interpolating the normalized neuronal activity to each stimulus condition. In practice, normalized neuronal responses were stored at sampling nodes in 12 separate $2 \mathrm{D}$ response matrices. We then interpolated the response matrices using a bicubic algorithm (MATLAB), which draws information from 16 neighboring measured values (their influence declining with cubic order of distance) to yield each interpolated value. [Matrices typically consisted of $11 \times 12$ (i.e., 132) measured values.] These response matrices were interpolated such that the resolution of the activity map increased from $200 \times 350 \mu \mathrm{m}$ (the measured resolution) to $10 \times 10 \mu \mathrm{m}$. The interpolated response matrices were plotted in gray scale and are analogous to the single-condition maps of neuronal activity commonly rendered by optical imaging or 2-deoxyglucose techniques.

Vector summation of interpolated activity matrices. To obtain 2D maps of preferred direction of motion from the conditional activity matrices, we adopted a procedure based on the algorithm used by Blasdel (1992) to extract orientation preference maps from V1 optical images. This procedure, which is widely used in optical imaging studies, facilitates comparisons between our results and published directional maps derived from intrinsic optical signals (Malonek et al., 1994; Shmuel and Grinvald, 1996; Weliky et al., 1996). Briefly, each of the 12 conditional activity matrices was multiplied by a unit vector, the direction of which corresponded to the stimulus motion used to obtain the measured neuronal responses for that matrix. These multiplications yielded a new set of 12 matrices, in which each element was a vector of direction corresponding to the stimulus motion and of length proportional to firing rate (measured or interpolated) at that map location. Finally, a matrix representing preferred direction at each map location was obtained by summing the 12 vectorial matrices. The direction and length of the resulting vector sum at each map location reflect, respectively, the neuronal preferred direction and strength of selectivity at that location. This vector-sum matrix was used to produce a $2 \mathrm{D}$ map of directionality, in which the preferred direction at each map location was represented by a color code.

To represent the strength of directional selectivity, we superimposed a lattice of arrows on the color-coded map. The lattice of arrows was a subsampled representation of the interpolated vector-sum matrix. The direction of each arrow was redundant with the underlying color map of directional preference; the strength of selectivity was uniquely conveyed in the map by arrow length. The resolution of the arrow lattice was selected to convey information in an optimal visual form and was limited simply by the need to avoid overlapping arrows.

Bootstrap algorithm for assessing reliability of neuronal responses. The length of each vector in the vector-sum matrix represents the strength of directional selectivity at the corresponding location. As noted above (see Statistical analysis), however, neuronal responsivity and directional selectivity measures were assessed for significance at each recording site. The results of these tests suggested that the vector-sum representation of directionality was less reliable at some map locations than at others. In practice, a given vector could be unreliable either because neuronal responses were highly variable across trials or because directional selectivity was poor (as manifested by pandirectional responses). Moreover, although unreliability was often associated with smaller vectors, this was not necessarily the case. For example, a large vector may result from the summation of highly variable responses, and conversely, a small vector may result from the summation of small but highly consistent responses. In addition, a small vector may result from the summation of large and consistent responses that are bidirectional.

As a result of these considerations, we sought a means to dissociate vector magnitude from vector reliability. The method we chose was based on a bootstrap algorithm, which involved recalculating the set of 12 interpolated activity matrices and the vector-sum matrix a total of 300 times for each map. Each iteration of this procedure differed only in that the set of trials $(n=10)$ used to compute the average neuronal response at each recording site was selected randomly, with replacement, from the real set of trials. Because the random-with-replacement procedure allows the activity on any given trial to be overrepresented in the average, the probability of obtaining a different average (and hence a different activity matrix) on each iteration is related to intertrial variability. It follows that, when the intertrial variability of measured neuronal responses is high, the bootstrap vector-sum matrix can be very different on successive iterations. This procedure is also highly sensitive to vector-sum unreliability associated with pandirection recordings: because pandirectional sites present similar responses to all tested directions, a small change in only one of the conditional activity matrices at the corresponding site, brought about by the random trial selection, will lead to a different bootstrap vector-sum estimate of the preferred direction and strength of selectivity at that map location.

Reliability at each location in the real vector-sum matrix was thus proportional to the SE across 300 bootstrap vector-sum matrices. To visually convey this measure of reliability in the color-coded directional maps, we assigned the brightness of each color to be inversely proportional to the SE matrix derived from the bootstrap procedure. Dark regions represent areas with low reliability or pandirectional cells.

Identification of fractures and singularities. Both types of representational discontinuities - fractures and singularities — documented previously for other cortical areas were identified in our quantitative MT data set by applying a 2D gradient operator to the interpolated maps of preferred direction (Shmuel and Grinvald, 1996). Discontinuities were defined as map locations for which the gradient of preferred direction exceeded the map-averaged gradient by at least a factor of 2. Fractures were also distinguished from singularities on visual inspection by the presence of linear (1D) versus punctiform (2D) qualities, as well as the presence of radial (pinwheel) arrangements of directional preferences surrounding singularities. On some occasions, we were able to detect a distinct architectural feature that consisted of two half-rotation $\left(180^{\circ}\right)$ singularities linked by a fracture.

Statistical estimation of the interpolation precision. Our interpolation procedure yielded a map of preferred direction at a resolution of $10 \times 10$ $\mu \mathrm{m}$. The precision of the interpolated values is limited by the sampling resolution and is expected to decline with distance from sampled points 

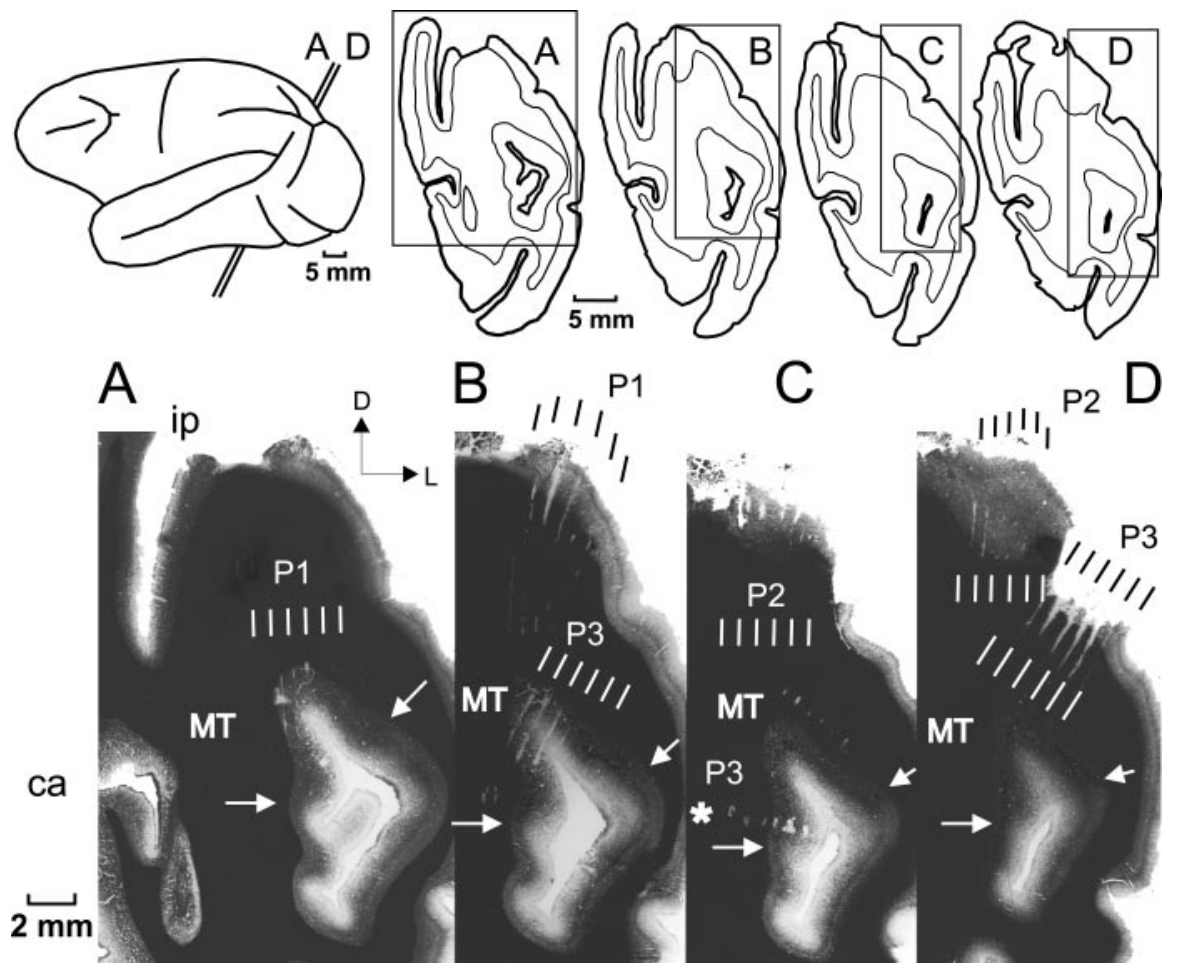

Figure 2. Representative data for reconstruction of electrode array penetrations from one animal (monkey B) that was studied via qualitative RF measurements. Inset, Top left, A lateral view of the brain along with the angle ( $28^{\circ}$ posterior to the frontal plane) and positions of the serial sections from which electrode penetrations were reconstructed. Scale bar, $5 \mathrm{~mm}$. Insets, Top right, Tracings of four representative serial sections are shown at the top right for reference. Scale bar, $5 \mathrm{~mm}$. The portion of each section highlighted by a rectangle is illustrated as a photomicrograph at bottom. $A-D$, These sections were spaced at $0.4 \mathrm{~mm}$ intervals and stained for myelin using the Gallyas (1979) method. The ring of cortical tissue in the bottom right of each photomicrograph is the lower posterior extent of the superior temporal sulcus, which appears as an invagination in this plane of section. Area MT can be identified by dense myelination along the upper portion of this ring of cortical tissue; the boundaries are indicated by arrows. Three microelectrode array penetrations were made in this animal in a plane approximately parallel to the plane of section. Two of these penetrations (P1 and P2) entered from the dorsal margin of the sections; the third penetration (P3) entered from the dorsolateral margin ( $30^{\circ}$ lateral to the sagittal plane). Gliosis caused by each penetration appears at different dorsoventral levels in different sections because of a slight difference between the angle of penetration and the angle of section. The general trajectories of the three penetrations are indicated by white and black bars. One identified electrolytic lesion is indicated by an asterisk in C. Complete penetrations reconstructed from the full set of histological sections were used to identify the locations of all MT recording sites, which in turn were projected onto the cortical surface of area MT. (nota bene, Not all penetration landmarks are visible in the low-power photomicrographs used for this illustration.) Scale bar, $2 \mathrm{~mm}$. ip, Intraparietal sulcus; ca, calcarine sulcus; D, dorsal; L, lateral. (See Fig. 7.)

in the matrix. To evaluate the interpolation error at different map positions, we applied a method inspired by Swindale et al. (1987). The method is based on comparison of interpolated and measured values of preferred direction at sampled map locations (i.e., recording sites). The basic idea is to estimate the precision of the interpolation at each nonsampled position, based on the precision of reinterpolated values at sampled locations that have been derived from interpolated values at nonsampled positions. The premise is that the precision of the interpolation procedure is similar regardless of whether one interpolates values at nonsampled positions using sampled map values or whether one reinterpolates values at sampled map locations using interpolated values from nonsampled positions.

To obtain interpolated values at sampled locations we performed the interpolation twice. First, the interpolated values of single-condition maps were obtained for nonsampled locations corresponding to the matrix shifted relative to the original matrix by a $2 \mathrm{D}$ vector. Second, new values of single-condition maps were obtained at the sampled locations by interpolating back from the shifted locations to the original locations. The new, twice-interpolated values of preferred direction at the sampled locations were then compared with measured values. The SD of the distribution of the difference between measured preferred directions and the interpolated one, divided by the square root of 2 (because the estima-

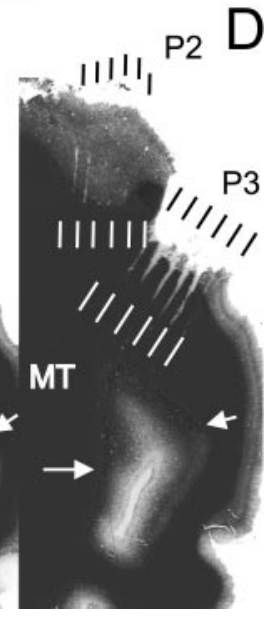

tion of error involved two acts of interpolation), is a statistical estimation of the interpolation error. We evaluated this error measure as a function of the map displacement vector defined above.

Inhibitory versus excitatory influences on neuronal responses. Initial evaluation of $2 \mathrm{D}$ interpolated maps suggested a relationship between (1) the relative influences of inhibition and excitation on directional tuning and (2) the location of the recording site relative to discontinuities. To quantify this relationship, we adopted an index of relative excitation and inhibition, which we termed "index of inhibition." This index was calculated (for monkeys $\mathrm{D}$ and $\mathrm{E}$ only) by the equation: $I=-($ Resp $S p) /(\operatorname{Resp}+S p)$, where Resp is the neuronal response at a given recording site averaged over trials and stimulus conditions, and $S p$ is the average spontaneous activity at the recording site. The index can assume values between -1.0 and 1.0. Positive values occur when the average inhibitory contribution to directional tuning is larger than the average excitatory contribution. Conversely, negative values occur when the average inhibitory contribution is smaller than the average excitatory contribution.

\section{Results}

A total of 1570 multiunit recording sites were studied in tangential multielectrode penetrations through area MT in five animals. Of these, 985 were studied in monkeys A, B, and C by qualitative assessment of RF properties, which involved manual presentation of moving bars and the experimenter's judgments of neuronal responses reproduced on an audio monitor. The remaining 585 recording sites were studied in monkeys D and E by quantitative characterization of RF properties.

Among the neuronal recording sites in the qualitative sample, $79 \%$ responded to moving stimuli. Ninety-three percent of the responsive sites, in turn, exhibited directional selectivity; the remaining responsive sites were pandirectional. Among the neuronal recording sites in the quantitative sample, we found $79 \%$ to be responsive to moving gratings and $84 \%$ responsive to moving dot arrays. Eighty-five percent of the responsive recording sites exhibited directional selectivity for gratings and/or dots. The remaining 15\% were pandirectional.

\section{Recording site localization}

Figures 2 and 3 present histological data used for the reconstruction of multielectrode array penetrations through the cerebral cortex. Shown are data from two animals, one of which (monkey $B$ ) is representative of the 6-electrode array experiments and the other (monkey E) representative of the 11-electrode array experiments.

Figure 2 (monkey B) contains portions of four sections that include area MT. These sections (Fig. $2 A-D$, inset) progress from anterior to posterior levels, and they present evidence for the locations of three penetrations (P1, P2, and P3) of the sixelectrode array (angled $28^{\circ}$ posteriorally from the frontal plane). The plane of the section does not correspond precisely to the 
plane of the electrode tracks, and thus each electrode array penetration traversed only a portion of a given section. The superior temporal sulcus appears as an island of cortical tissue in each of these sections. Area MT (identified by dense myelin staining) lies in the dorsal portion of this island and is bounded by two arrows superimposed on each section in Figure 2.

Gliosis from the first penetration (P1) can be seen in Figure $2 A$ at the dorsal margin of the superior temporal sulcus in the region of area MT. P1 also appears in Figure $2 B$ (angled slightly because of differential tissue shrinkage) at the dorsal surface of the brain. Evidence for the second penetration (P2) can be seen in Figure 2, C and $D$. Portions of the third penetration (P3), which entered the brain obliquely from the dorsolateral surface (angled $30^{\circ}$ from the sagittal plane), can be seen at the cortical surface in Figure $2 D$, in white matter in $C$, and at the level of MT in B. An electrolytic lesion made at the end of P3 can be seen in $C$.

Figure $3 A-D$ (monkey E) also contains portions of four sections that progress from anterior to posterior levels. These sections present evidence for the locations of three penetrations (P1, P2, and P3) of the 11-electrode array, all of which progressed along an oblique dorsoventral trajectory (angled $12^{\circ}$ posteriorally from the frontal plane) through the brain. Each electrode array traversed only a portion of a given section. Evidence for the first penetration (P1) is present at the dorsal cortical surface in Figure $3 A$. Similarly, evidence for the second penetration can be seen in $B$ and $C$. The progression of the third penetration (P3) is visible at the level of MT in $D$.

\section{General character of neuronal responses to visual motion}

\section{Representative responses}

Directional tuning in area MT of monkeys D and E was assessed quantitatively using computer-controlled visual stimulation and data acquisition/analysis (see Materials and Methods). The responses to stimulus motion observed at a typical multiunit recording site (monkey E) are illustrated in Figure 4. Around the perimeter of the figure are two sets of peristimulus histograms, which illustrate responses elicited by moving gratings (gray) and dot patterns (black). Either stimulus moved in 12 different directions, which correspond to the angular positions of the histograms in Figure 4. For each stimulus type and direction, the mean firing rate elicited during the period of moving stimulus presentation (indicated by the bar under each histogram) was plotted on the polar axes at the center of Figure 4.

As is characteristic of area MT, these responses revealed a high degree of unidirectional selectivity. This multiunit clearly exhibited stronger responses to moving dots than to gratings. Directional tuning bandwidth for gratings $\left(159^{\circ}\right)$ was slightly larger than that to random dot fields $\left(142^{\circ}\right)$. Directionality of neuronal responses, assessed using a standard directionality index (see Ma- terials and Methods), was similar for gratings (1.29) and dots (0.99), despite the observed differences in the response magnitudes and tuning bandwidths. No significant responses were seen for static presentations of the stimuli, which preceded stimulus motion on each trial.

\section{Population statistics}

At each recording site in monkeys $\mathrm{D}$ and $\mathrm{E}$, we obtained data in the format shown in Figure 4. These data formed the basis for neuronal activity maps that were used to generate 2D maps of directionality (see below), and they were used to characterize the behavior of the population of area MT neurons studied. To accomplish the latter, we first fitted Gaussian functions to data such as those in Figure 4. The parameters of these functions were then used to compute important tuning metrics, such as directional bandwidth, index of directional selectivity, and preferred direction of motion. The distributions of these metrics for the population of recorded units are plotted in Figure 5, $A, B$, and $C$, respectively.

For clarity of illustration, the population distributions of directional tuning bandwidth (Fig. 5A) and directionality index ( $B$ ) are shown only for responses to moving gratings. The mean $\left(104^{\circ}\right)$ and $\mathrm{SD}\left(29^{\circ}\right)$ of the bandwidth distribution are similar to values reported previously for macaque area MT ( 91 and $35^{\circ}$ ) (Albright, 1984). The mean of the directionality index distribution was 0.95 , which is slightly smaller than the value reported previously for macaque MT (1.00) (Albright, 1984). However, the macaque data were obtained from single-unit recordings; the smaller directional index values seen in Cebus may reflect the 


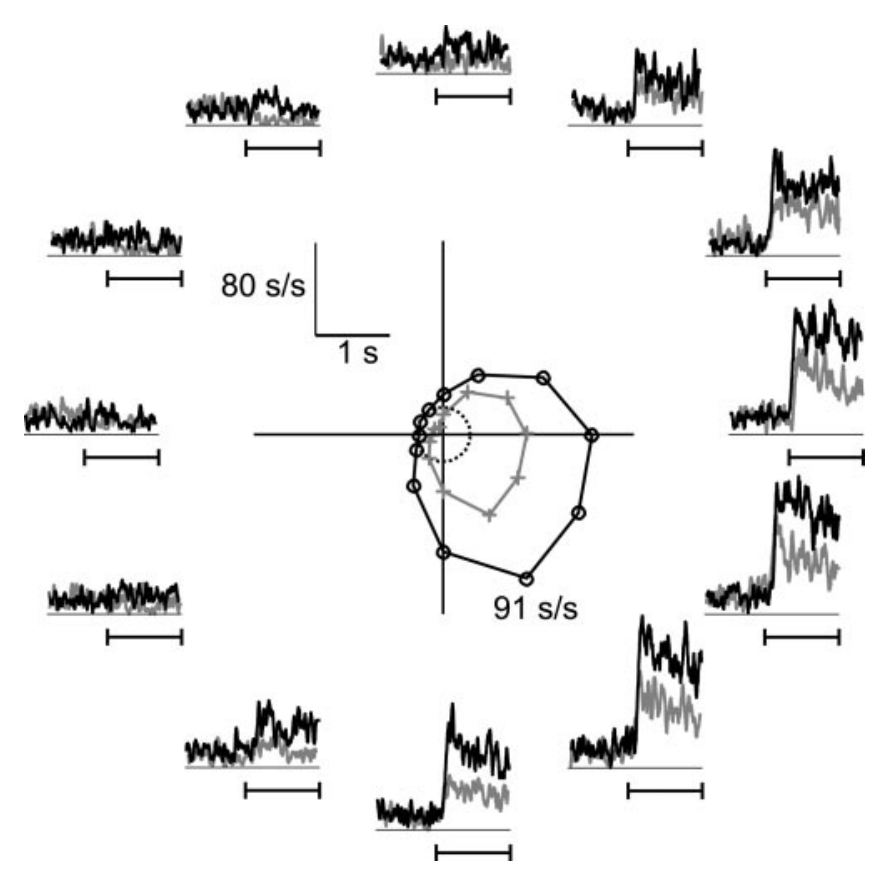

Figure 4. Neuronal activity elicited from a typical unidirectional multiunit cluster in area MT. The RF was located in the lower visual field quadrant contralateral to the recorded hemisphere and within $10^{\circ}$ of the center of gaze. Visual stimuli consisted of gratings and dot patterns that were each moved within the RF in each of 12 different directions. Peristimulus response histograms for gratings (gray) and dots (black) are plotted around the perimeter of the figure with azimuth corresponding to the direction of motion. The bar under each histogram indicates the period of time in which the stimulus was moving in the RF. Individual histograms represent responses summed over 10 stimulus presentations. The polar graph at center represents the mean response rate for each direction, plotted separately for gratings (gray) and dots (black). The dashed circle indicates the level of spontaneous activity. Responses to gratings were characteristically weaker than those to moving dot patterns. Directional tuning bandwidths and indices of directionality were similar for the two stimulus types. $\mathrm{s} / \mathrm{s}$, Spikes per second; $1 \mathrm{~s}, 1 \mathrm{sec}$.

occasional (and undetectable in post hoc analyses) inclusion in multiunit recordings of neurons with opposing directional preferences.

The distribution of preferred directions across the population of recording sites appears in Figure $5 C$. This distribution contains values of preferred direction for all recording sites at which a reliable estimate could be obtained. Hand-mapped estimates of preferred direction are known to be similar (within $45^{\circ}$ ) to computer-mapped estimates (Albright, 1989), provided that responses are strong, and we have consequently pooled all reliable estimates from monkeys A to E. The resulting distribution exhibits a small but significant $\left(\chi^{2}=20.3\right.$; $\left.\mathrm{df}=11 ; p<0.041\right)$ bias centered on $\sim 180^{\circ}$ (downward motion). Interestingly, a similar trend (albeit not statistically significant) can be seen in previously published data from macaque MT (Albright et al., 1984; Albright, 1989).

Finally, Figure $5 D$ illustrates the distribution of changes in preferred direction seen between all pairs of successive recording sites (sampled every $200 \mu \mathrm{m}$ ) for which reliable estimates could be obtained (again pooling data from monkeys A to E). For most pairs of sites, the preferred direction difference was between 0 and $60^{\circ}$, although a smaller peak in the distribution appears between 135 and $180^{\circ}$. The former mode indicates that preferred direction typically changed in a gradual manner on each electrode as the electrode array traversed across the cortex of area MT. The latter mode reflects abrupt reversals in preferred direction, which typically occurred at fractures or singularities in the direction map (see below). This combination of smooth and abrupt changes between recording sites is very similar to that originally reported for the macaque MT (Albright et al., 1984).

Comparison of responses with gratings and dot patterns

The multiunit shown in Figure 4 exhibited responses to moving dot stimuli that were stronger and more narrowly tuned than were responses to moving gratings, although the preferred direction remained the same. To determine whether these differences and similarities reflected a general tendency, we compared differential response magnitudes, tuning bandwidths, indices of directionality, and preferred directions for grating and dot stimuli across the population of neurons for which reliable measurements could be made using both stimulus types. Comparing sites with significant responses to dots and gratings, we found that the stimulus type used significantly influenced the first two of these measures: on average, relative to gratings, the responses to dot stimuli were larger (dots, $27 \mathrm{sec} / \mathrm{sec}$; gratings, $19 \mathrm{sec} / \mathrm{sec} ; p<$ 0.0001 ; paired $t$ test) and more broadly tuned (dots, $121^{\circ}$; gratings, $105^{\circ} ; p<0.001$; paired $t$ test). Directional indices for the two stimulus types did not differ (dots, 1.05 ; gratings, $1.03 ; p>0.623$; paired $t$ test), however. These effects indicate that area MT of the Cebus monkey possesses some degree of sensitivity to stimulus form, which is a point that we will address in detail in a forthcoming report.

For the purposes of the present study, it was most important to know whether there were any significant differences between the preferred directions detected using grating versus dot stimuli. Such differences, should they exist, would imply the existence of different 2D directionality maps for the two stimulus types and would thus foil arguments regarding the generality of any maps that we observed. To address this issue, we cross-plotted the preferred directions observed using gratings versus dots (Fig. 6), and we assessed the relationship between the two measures by circular correlation (Batschelet, 1981). The result indicates that the preferred directions were similar under these two stimulus conditions and significantly correlated [circular correlation coefficient, $\left.r=0.632\left(r^{2}=0.405 ; p<0.001\right)\right]$. These results led us to expect a high degree of similarity between $2 \mathrm{D}$ directionality maps for the two stimulus types. Our observations supported this prediction (see below).

\section{Discrete functional maps derived from qualitative RF measurements}

Two-dimensional maps of axis-of-motion and direction-ofmotion preference are illustrated in Figures 7 and 8, respectively. The illustrated maps were derived from hand-mapped measures of directional preference at each recording site along three multielectrode penetrations made in monkey B. Visualization of ordered arrangements in area MT was often facilitated by examining preferred axis of motion (computed directly from preferred direction; see Materials and Methods), rather than preferred direction of motion, because abrupt directional reversals were removed (Albright et al., 1984). We thus begin our description of these maps by considering the $2 \mathrm{D}$ representation of preferred axis of motion.

\section{Maps of preferred axis of motion}

Figure 7 contains four axis-of-motion maps obtained from area MT of monkey B, which are representative of our findings from hand-mapped RFs. Three of these maps (Fig. 7A-C) were derived from multiunit RF measurements made along three of the six electrode array penetrations (P1, P2, and P3) that were taken in 
this animal. The fourth map (Fig. 7D) covers a patch of cortex that is represented in both $B$ and $C$, but it is a composite derived from the fortuitous overlap of two array penetrations (P2 and $\mathrm{P} 3$ ). (The P2 data in Figure $7 D$ are clipped from a portion of $B$, indicated by the dotted line. The P3 data in Figure 7, $C$ and $D$, are spatially coextensive and identical.) The locations of the three array penetrations relative to sulcal topography are indicated in Figure $7 E$. Penetrations passing through area MT were approximately parallel $\left(10-12^{\circ}\right)$ to the surface of area MT. Preferred axis-ofmotion values at each recording site have thus been projected directly onto a flat rectilinear representation of the cortical surface in each map and are indicated by oriented line segments. Significant map features revealed by comparison of selectivities along and between electrodes are highlighted in each map by elongated rectangles and ellipses/circles, respectively.

The map in Figure $7 \mathrm{~A}$, which was derived from the first array penetration (P1), contains linear sequences (L1, L2, and L3) from three adjacent electrodes in which the preferred axis of motion rotated gradually through $180^{\circ}$ as the electrodes traversed 1-2 mm of cortex. This type of sequence regularity closely matches that seen along single electrode penetrations in macaque MT (Albright et al., 1984). We hypothesized that there may be additional map features, radial or pinwheel configurations, that were undetectable from any single electrode. In an initial attempt to evaluate this hypothesis, we examined preferences both within and between adjacent penetrations for hints of radial symmetry. In the region delimited by the ellipse (R1), for example, diametrically opposed recording sites exhibited roughly parallel axis-ofmotion selectivity. This arrangement is consistent with a pinwheel formation having a diameter of $\sim 1.4 \mathrm{~mm}$.

The map illustrated in Figure $7 B$ was derived solely from the second array penetration (P2) and is notable for the nearly uninterrupted samples from four of the six electrodes over a $7 \mathrm{~mm}$ extent. From these four electrodes and a fifth, we identified five sequences of preferred axis of motion that cycled through $\sim 180^{\circ}$ (L5, L6, L8, L10, and L11) and three sequences of $\sim 90^{\circ}$ (L4, L7, and L9). Along this penetration we also observed a great deal of variability in the lengths of sequences $\left(180^{\circ}\right.$ cycle, $1-1.8 \mathrm{~mm} ; 90^{\circ}$ cycle, $0.8-1.4 \mathrm{~mm}$ ), which may reflect different angles of intersection with cortical modules for motion processing (Albright et al., 1984). Radial patterns of preferred axis of motion (R2-R4) not detectable from any single electrode also emerged from integration of sequences along adjacent pairs and triplets of electrodes.

The map illustrated in Figure $7 C$, which was derived from the third array penetration (P3), also contains notable sequences of regular linear progression (L12-L16), two of which cycle $180^{\circ}$ or more (L14 and L15). Comparisons between adjacent electrodes suggest radial arrangements that encompass a full cycle of $180^{\circ}$ (R5 and R6).
As shown in Figure $7 E$, the second array penetration (P2) happened to traverse a region of area MT that was also mapped by P3. The combined data set representing the intersection of these two arrays is shown in Figure $7 D$. Although we must assume a small amount of error in the alignment of reconstructed arrays, recording sites at their points of intersection exhibited remarkably consistent preferences (e.g., the three points of intersection of P2 with sequence L14 of P3). More generally, the linear sequences (L12-L16) and radial patterns (R5 and R6) identifiable from the P3 data set received confirmation from P2. Some additional features, such as the broad radial pattern R7, which was not confidently recognizable in either data set considered alone, emerged from the P2 plus P3 map.

\section{Maps of preferred direction of motion}

Figure 8 contains four direction-of-motion maps derived from the same array penetrations and recording sites that were presented in Figure 7 to illustrate regularity of axis of motion. The preferred direction of motion observed at each site is represented by an arrow. To facilitate comparisons between axis- and direction-of-motion maps, the locations of salient features (linear sequences and pinwheels) that were identified in the axis-ofmotion maps (highlighted in Fig. 7) are also indicated in Figure 8. The preferred direction maps reveal that gradual sequences of preferred axis of motion are commonly interspersed with $180^{\circ}$ reversals in preferred direction of motion, a finding that is consistent with previous observations from macaque MT (Albright 


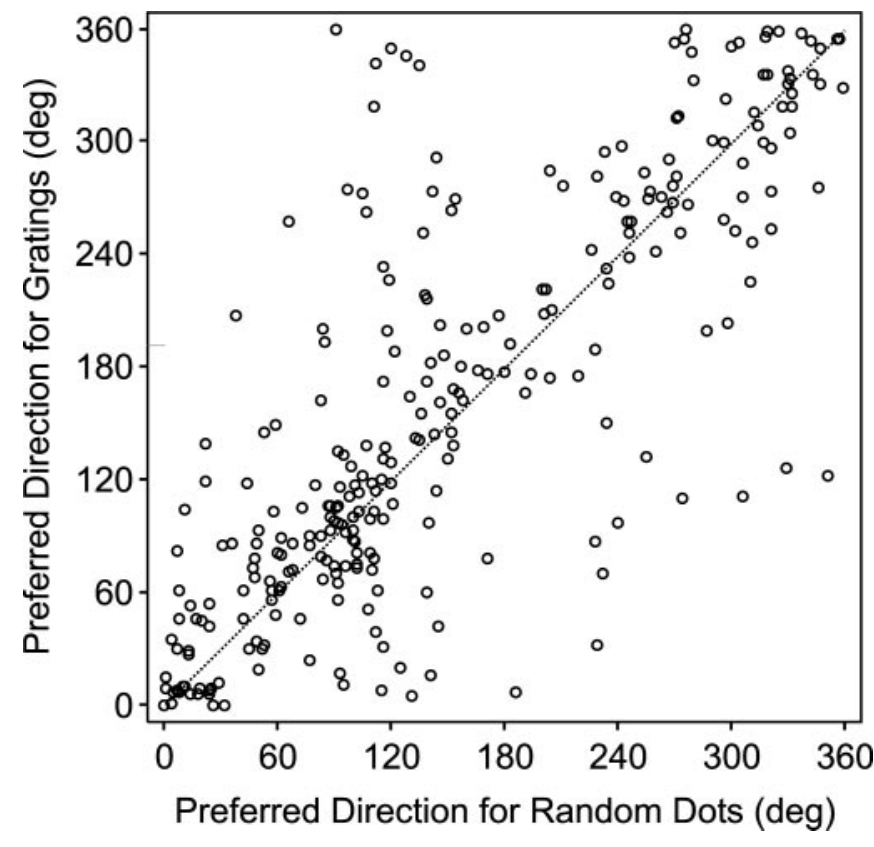

Figure 6. Comparison of preferred directions of motion determined using moving gratings versus moving dots. Each point in the scatter plot represents the grating ( $y$-axis) and dot $(x$-axis) preferred directions for one recording site. The plot contains data from all sites for which both measures could be determined reliably $(n=292)$. A line with unit slope is plotted for reference. The proximity of data points to this line indicates that the two measures were similar for most recording sites and were highly correlated (circular correlation coefficient $=0.405$; $p<0.02$ ).

et al., 1984). Adjacent recording sites that exhibited such $180^{\circ}$ directional discontinuities are indicated by red arrows in Figure 8. One significant consequence of these frequent directional discontinuities, detectable on close examination of the maps, is the complete absence of continuous sequences spanning $360^{\circ}$ or more.

\section{Continuous functional maps derived from quantitative RF measurements}

Using computer-controlled visual stimulation and data acquisition methods, we assessed the directional preference at each recording site along multielectrode penetrations in monkeys D and E. Although these measurements, as for our hand-mapped RFs, were made at discrete positions within a lattice parallel to the cortical surface, the quantitative characterization of directional tuning at each recording site enabled us to reliably interpolate tuning values between sites. This interpolation (see Materials and Methods) yielded 2D maps that can be readily compared with functional maps obtained by optical recording (Blasdel and Salama, 1986; Ts'o et al., 1990; Malonek et al., 1994; Shmuel and Grinvald, 1996; Weliky et al., 1996). We present these maps in two forms: (1) single condition maps, which individually represent the pattern of activity elicited by a single direction of motion, and (2) composite condition maps, which integrate the patterns of responses across all directions of motion and indicate via a color code the directional preferences at each map coordinate.

Single-condition maps of neuronal responses

Figure 9 illustrates a single-direction $\left(0^{\circ}\right.$; rightward $)$ interpolated firing rate (normalized per recording site) map derived from one 11 -electrode array penetration made in monkey E. The rectangular grid represents a $2 \mathrm{D}$ expanse of tissue within area MT, parallel to the cortical surface. Stimulus selectivities were assessed at the points indicated by crosshairs, which define an $11 \times 12$ sampling matrix with a resolution of $350 \times 200 \mu \mathrm{m}$. Using the bicubic interpolation procedures described in Materials and Methods, the activity map was interpolated to a resolution of $10 \times 10 \mu \mathrm{m}$.

Figure 10 shows a set of 12 single-condition maps, which represent the pattern of normalized responses for each stimulus direction used. The map in the top left corner $\left(0^{\circ}\right)$ is replotted from Figure 9. To convey the relationship between these maps and the neuronal responses on which they are based, we have paired each map with a peristimulus histogram that illustrates the neuronal response elicited by the moving grating stimulus at the map location indicated by the crosshair. The recording site illustrated was unidirectional. The preferred direction of motion was determined to be $129^{\circ}$ (up and left), directional tuning bandwidth was $108^{\circ}$, directionality index was 0.98 , and differential response magnitude was 85 spikes/sec.

Comparison of two single-condition maps generated from stimuli moving in opposite directions (e.g., $0^{\circ}$ and $180^{\circ}$ ) reveals that oppositely moving stimuli elicited similar activity levels from some map regions and different activity levels from others. Conditions such as this, in which contrasting stimulus conditions elicit both similar and different activity levels at different map locations, also occur in optical imaging data (Malonek et al., 1994; Shmuel and Grinvald, 1996; Weliky et al., 1996). There are multiple potential explanations for such effects. For example, similar activity patterns may reflect clusters of bidirectional neurons, or they may be indicative of regions in which oppositely preferring unidirectional neurons are intermixed. Unlike intrinsic optical data, our multielectrode approach permits cellularresolution access to the sources of the interpolated signals and may thereby elucidate mechanisms of cortical processing. As shown in Figure 10, for example, preferred $\left(120^{\circ}\right)$ and opposite $\left(300^{\circ}\right)$ directions yielded different map activations at a unidirectional recording site. Indeed, provided that the opposing stimulus pairs elicited different neuronal responses (as for $0 / 180^{\circ}, 90 /$ $270^{\circ}, 120 / 300^{\circ}$, and $150 / 330^{\circ}$ ), the maps were necessarily different at the location of the recording site. If the recording site was either bidirectional or pandirectional, however, map activity levels for opposing stimulus pairs would be similar. In addition, map regions may exhibit similar activity levels simply because cells at the recording site responded poorly to directions along that axis (as for $30 / 210^{\circ}$ ). The distribution of directional indices (Fig. $5 B$ ) from the sample of recorded units reveals that the recording sites were overwhelmingly unidirectional (74\%). This finding alone suggests that the activity levels (measured or interpolated) to pairs of opposite directions should be different in most regions of most maps.

\section{Composite maps of preferred direction}

Single-condition maps (Fig. 10) were obtained from every patch of area MT that was studied in monkeys D and E using the multielectrode array. A separate set of single-condition maps was obtained for each stimulus type used (gratings and dots). From these data, we derived separate composite maps of preferred direction (see Materials and Methods) for gratings and dots. One representative map of each type is presented in the following sections.

\section{Directional maps for moving gratings}

Figure $11 \mathrm{~A}$ illustrates a representative composite map of preferred direction for moving gratings. To convey the strength of directional selectivity across the map as well as the directional preference, arrows reflecting the full vector description (direction 

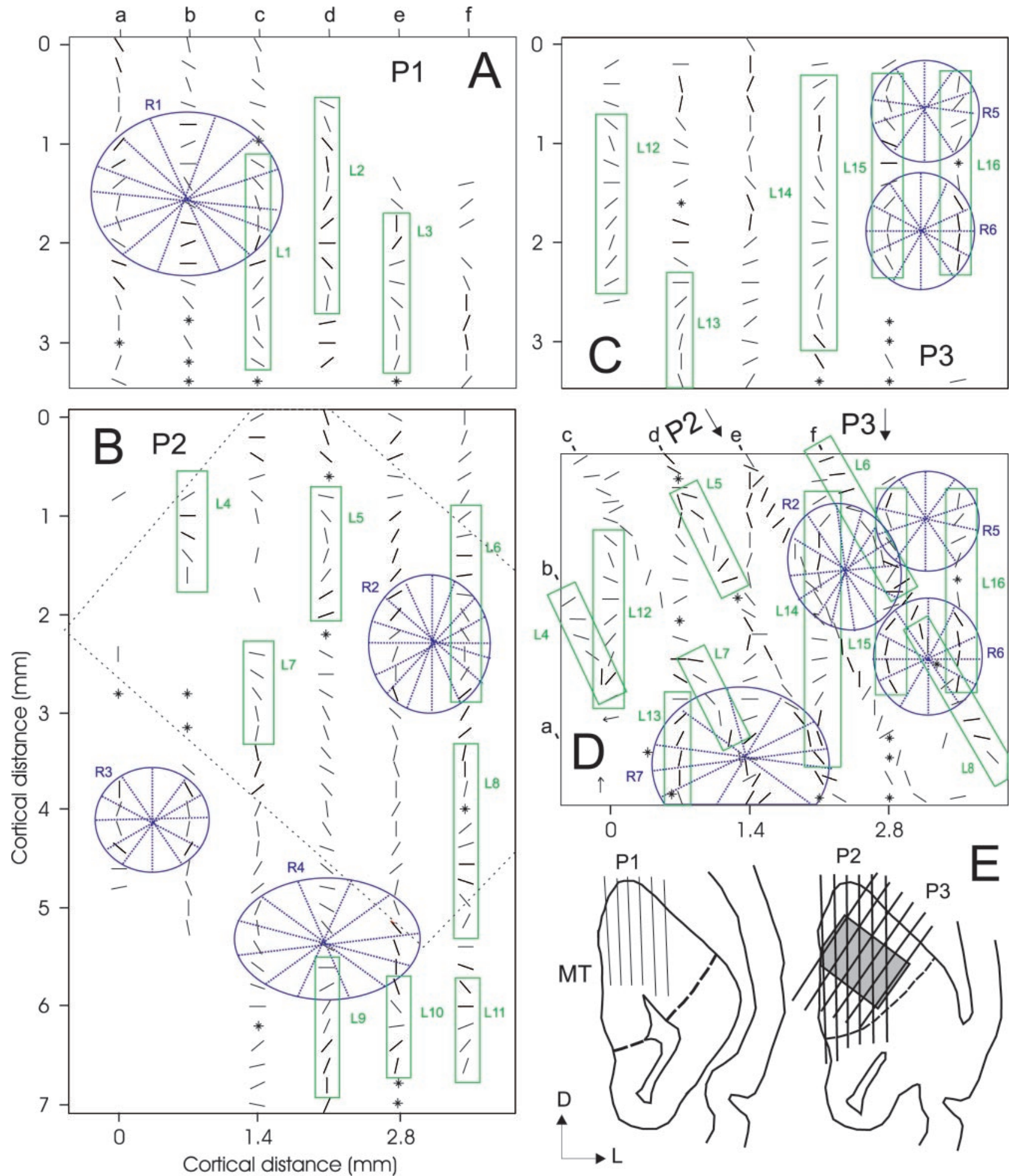

Figure 7. $A-D$, Two-dimensional maps of preferred axis of motion derived from qualitative RF measurements obtained along three multielectrode array penetrations (P1, $P 2$, and $P 3)$ in monkey $B$. E, Line drawings of serial sections sliced in the plane of array penetrations, on which the boundaries of MT and the paths of electrodes are indicated. The trajectories of these multielectrode penetrations are shown in greater detail in Figure 2.A, A rectangular expanse of MT parallel to the cortical surface, on which the locations of P1 recording sites have been projected. The six electrodes of the array (a-f) entered this rectangular panel (and all others, except where noted otherwise) from its upper margin at $700 \mu \mathrm{m}$ spacing. Preferred axis of motion at each recording site is indicated by a small bar. Elongated rectangles (green) highlight map features evident from each electrode considered individually, which consist of smooth progressions (e.g., L1, L2, L3) of preferred axis of motion (sequence regularity). Ellipses (blue) show radial arrangements (e.g., R1) or pinwheels that emerged from recording sites within and between adjacent electrodes. Stars indicate the locations of pandirectional recording sites. $B, C$, Data from arrays $\mathrm{P} 2$ and $\mathrm{P} 3$, respectively, and map features similar to those in $A . D, A$ region of cortical tissue through which $\mathrm{P} 2$ and $\mathrm{P} 3$ traversed with overlapping trajectories ( $E$, shaded rectangle). The P3 data in this panel are identical to those in C; the P2 data are drawn from the tilted rectangle (dotted line) in $B$. Recordings at regions of overlap between the two penetrations mostly corroborate selectivity measurements and map features, and they support the existence of additional map features that were not readily detectable from either data set alone. See also Figure 2. 

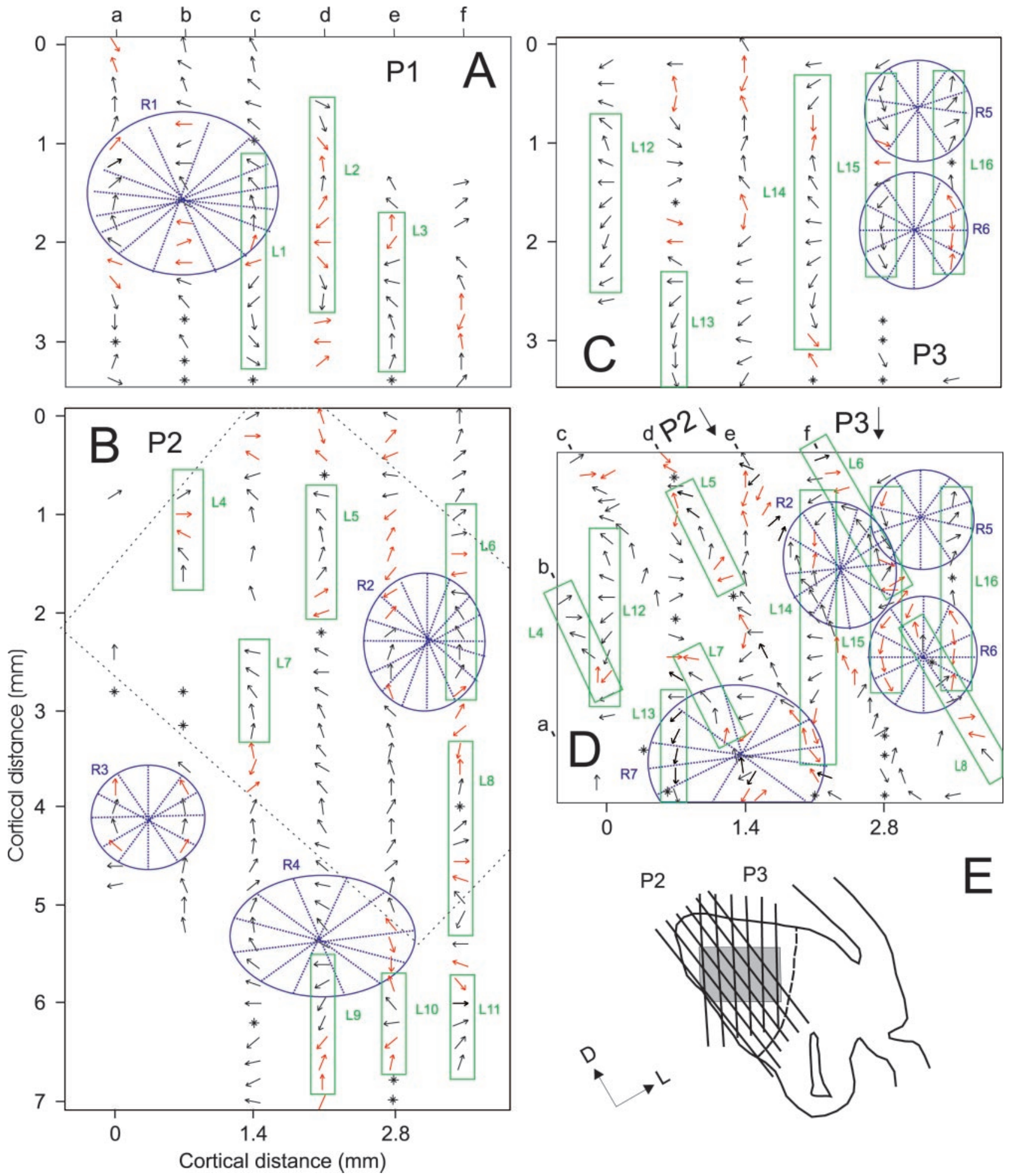

Figure 8. Two-dimensional maps of preferred direction of motion derived from qualitative RF measurements obtained along three multielectrode array penetrations (P1, P2, and P3) in monkey B. These data correspond to the same cortical regions and recording sites as those in Figure 7. All plotting conventions are the same as in Figure 7, except that preferred direction of motion is represented by the direction of a small arrow at each recording site. Red arrows are used to indicate the locations of pairs of recording sites for which preferred direction of motion shifted by $\sim 180^{\circ}$ (i.e., a directional reversal). E, The section from Figure 7E that illustrates the P2-P3 overlap. The section has been rotated counterclockwise to emphasize its relationship to the data in D. See also Figure 2 .

and length) at subsampled resolution have been overlaid on the color map. In most instances, arrow length bears a close relationship to the index of directionality, although very short arrows may reflect a high directionality index combined with very broad tuning (because 12 directions contribute to the vector sum). The range of arrow lengths displayed has been scaled to optimize illustration (i.e., to avoid overlap); it is only the relative lengths that convey the strength of directional selectivity. One conse- 
$\operatorname{Max}$

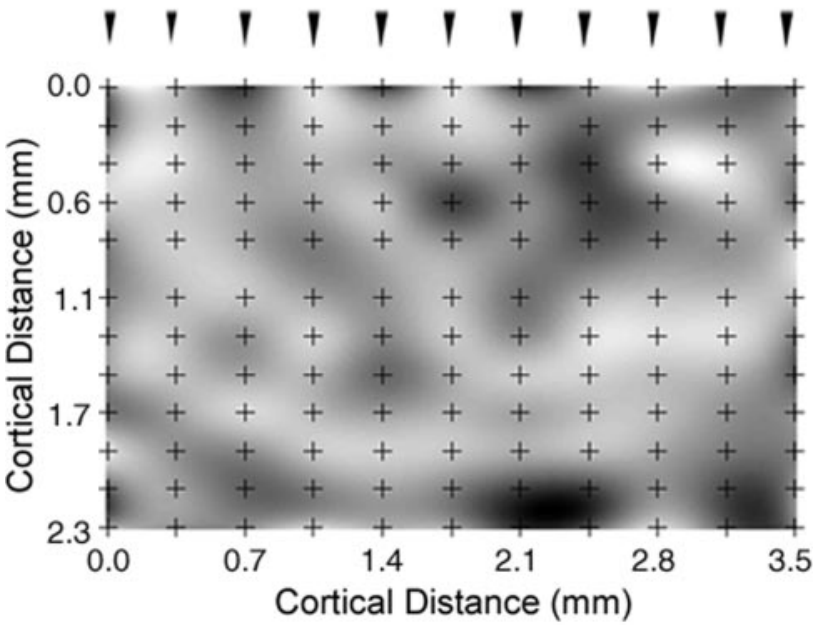

Figure 9. Single-condition map of normalized neuronal firing rates in the interpolated response matrix for a rectangular region of area MT in monkey E. Neuronal responses were sampled along a penetration of the 11-electrode array (see Materials and Methods). The stimulus was a grating that moved in direction $0^{\circ}$ (rightward). Small arrows at the top indicate trajectories of the 11 electrodes in the array. Crosshairs indicate actual recording sites. Normalized firing rates are proportional to gray-scale values. Max, Maximum firing rate; min, minimum firing rate.

quence of this scaling is that the arrows used to represent selectivity at significantly unidirectional map locations are sometimes quite small because of the presence of very large differential response magnitudes at one or more different map locations. As detailed in Materials and Methods, however, we have used a bootstrap procedure to dissociate the length of each vector from its reliability. Black regions in the composite map indicate areas in which the preferred direction and strength of selectivity could not be reliably ascertained because of a high degree of intertrial response variability or pandirectional recordings, or both.

The composite map in Figure $11 \mathrm{~A}$ is characterized by gradual changes in preferred direction that are interrupted by lines (fractures) and points (singularities), which fragment the continuoustoned areas into patches. Following the usage of Blasdel and Salama (1986) as applied to orientation preference maps in primary visual cortex, a directional fracture is a one-dimensional rift in directional continuity across the cortical surface, where the preferred direction changes by an amount that significantly exceeds the average. We identified these rifts systematically by examining the rate of change of preferred direction in composite maps. In practice, the rate of change was computed by applying the gradient transform to the composite maps (see Materials and Methods). Figure $11 B$ illustrates the gradient map derived from the directional map shown in $A$. Extended lines of high gradient (brighter areas) indicate fractures. Similarly, directional singularities occur at the termination of fractures and as isolated points of high gradient.

Figure $11 C$ highlights a directional fracture located in the top right quadrant of $A$, along with the neuronal responses that gave rise to the fracture. An elongated directional discontinuity extends obliquely from the bottom left to top right portions of the highlighted region and is most evident as the transition from red $\left(\sim 135^{\circ}\right)$ to green $\left(\sim 315^{\circ}\right)$. This discontinuity can also be seen in the gradient map of Figure $11 B$. The white crosshairs on the highlighted region (also shown on Fig. 11A) indicate the locations of recording sites that span the discontinuity. Polar plots of directional tuning recorded at these sites are shown outside the lateral margins of the map. [In these, and in all subsequent directional tuning plots, the maximum response rate (or spontaneous rate, if larger) has been normalized to the unit circle. The corresponding spike rate is indicated]. The plot on the left shows the tuning properties of the recording site illustrated in Figure 10. The plot at the bottom right is located on the opposite side of the fracture and is selective for the opposite direction. (nota bene, This evidence also documents the fact that the bicubic interpolation procedure of the firing rate does not eliminate directional fractures.)

The third recording site illustrated in Figure $11 C$ (top right) was located very close to the fracture, and the corresponding plot of directional tuning reveals two important properties: (1) the neuronal response was bidirectional, and (2) the response was primarily inhibited by moving stimuli, albeit differentially as a function of direction. Interestingly, the moving stimuli that elicited the strongest inhibition corresponded approximately to the preferred directions at the two sites highlighted above, which lie on opposite sides of the fracture. A survey of responses at all recording sites indicates that these response properties were commonly associated with fractures. Bidirectional sites at fractures were generally not very responsive, and their selectivity was often caused by inhibition. In addition, fractures were frequently populated by pandirectional recording sites (note dark regions along the fracture in Fig. 11C).

\section{Directional maps for moving dots}

Figure $12 \mathrm{~A}$ illustrates a representative composite map of preferred direction for moving dots. This map was derived from the same recording sites that were used for the moving-grating directional map of Figure 11, and the two maps thus represent different attributes from the same region of cortex. Our comparison of preferred directions for gratings and dots (Fig. 6) indicated a high degree of correspondence and led us to expect highly similar maps of preferred direction for these two stimuli. In accordance with this expectation, most portions of the illustrated maps for moving gratings (Fig. 11) and dots (Fig. 12) are similar, if not identical, in their selectivity patterns, and it is possible to identify many common architectural features, such as fractures, pinwheels, and slabs. A high degree of grating- versus dot-map similarity was also detected using data gathered on other penetrations. We have observed significant differences, however, between the effects of gratings and dots on response magnitude and directional tuning bandwidth (see above).

Figure $12 B$ highlights features of a band extracted from a central horizontal strip on the right side of the complete directional map in Figure 12A, along with the neuronal responses that gave rise to these features. The white crosshairs in Figure 12, $A$ and $B$, indicate the map locations where the illustrated neuronal responses were recorded. This band is instructive, because the rate of change of preferred direction varied significantly. Within some portions of the illustrated band, particularly the red-to-orange region on the left side of Figure $12 \mathrm{~B}$ and the blue-to-green region on the right side, preferred direction of motion changes gradually over a span of nearly $180^{\circ}$, in a manner similar to the sequence regularities detectable in the discrete directional map of Figure 8 (Albright et al., 1984). Other portions of the band, notably the narrow yellow strip on the left of Figure $12 \mathrm{~B}$ and the narrow purple strip in the center, contain rather abrupt shifts in preferred direction. By the criteria of Blasdel and Salama (1986) (see Materials and Methods), both of these shifts qualify as directional fractures. The shifts in preferred direction between the corre- 

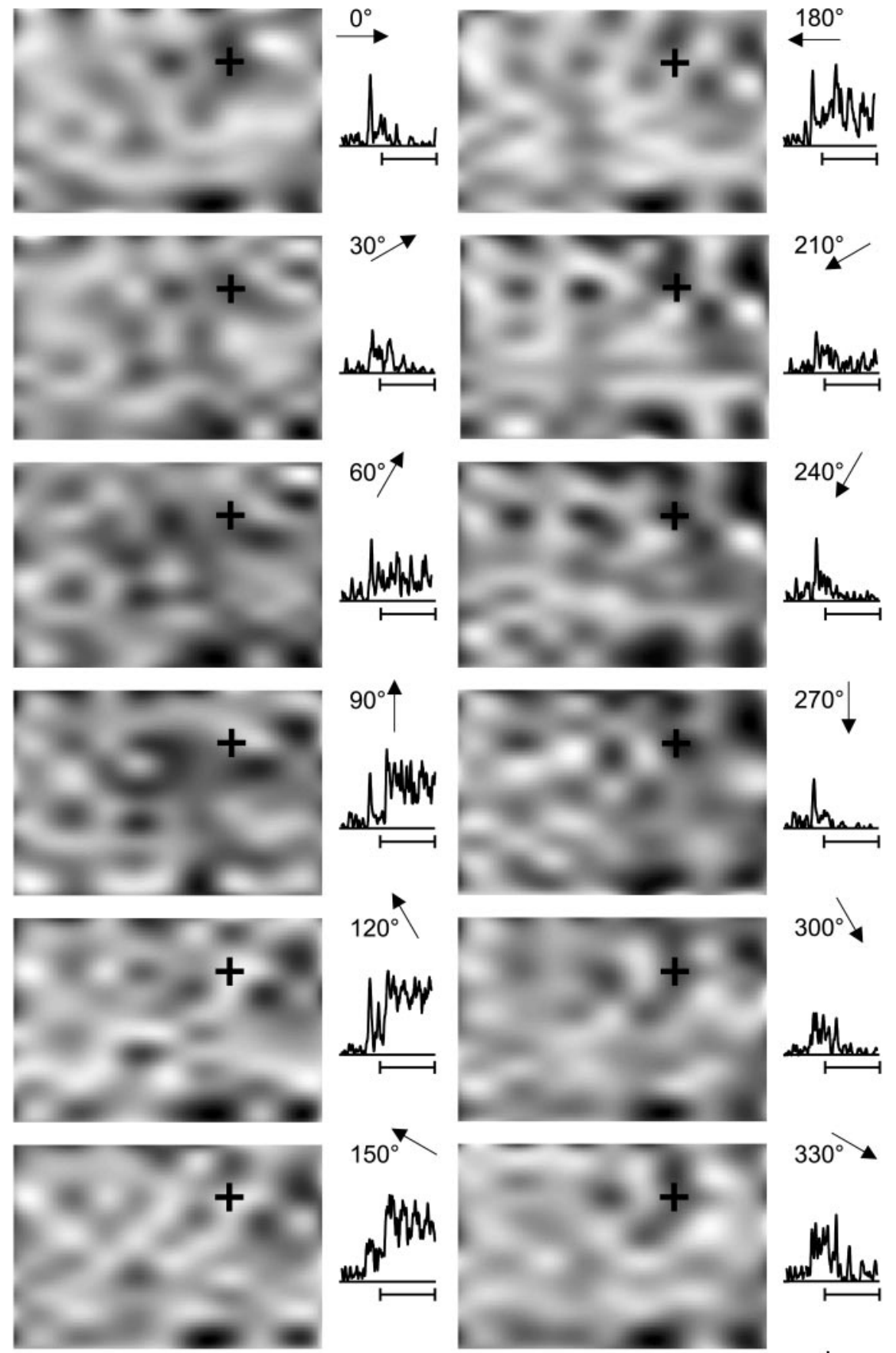

$\operatorname{Max}$
Min

$\mathrm{s} / \mathrm{s}$

$1 \mathrm{~s}$

Figure 10. Set of 12 single-condition maps, each of which represents normalized neuronal firing rate as a function of position in the same rectangular region of area MT. Each map presents firing rates elicited by 1 of the 12 different directions of motion of a grating within the RF. The map at top left (direction $0^{\circ}$ ) is the same as that shown in Figure 9. To the right of each map appears a peristimulus response histogram for a unidirectional multiunit that was recorded from the site indicated by the crosshair in each map. (As indicated in Materials and Methods, stimulus motion was preceded by a brief static presentation of the stimulus, which often elicited a transient neuronal response, as was the case for this recording site.) Min, Minimum firing rate; max, maximum firing rate; ss, spikes per second; $1 \mathrm{~s}, 1 \mathrm{sec}$.

sponding recording sites provide direct support for this interpretation. Interestingly, the neuronal responses recorded at the first and third sites in Figure $12 \mathrm{~B}$, which lie closest to the indicated fractures, exhibited a high degree of inhibition; indeed, the selectivity was wrought almost entirely by inhibition. Responses re- $180^{\circ}$

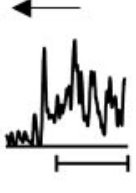

$210^{\circ}$
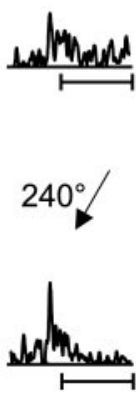

$270^{\circ}$
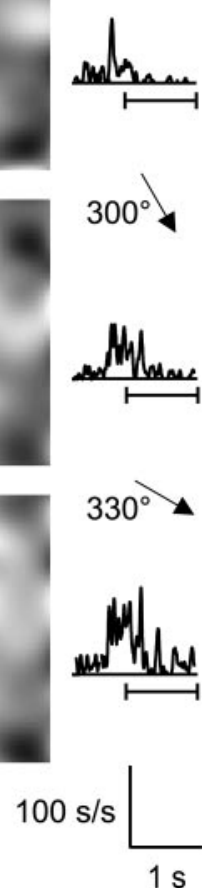

corded at the other sites illustrated in Figure $12 \mathrm{~B}$ exhibited more typical ratios of excitation versus inhibition. Such arrangements, in which highly selective sites manifesting excitatory responses and opposing directional preferences are separated by a site in which inhibition is predominant, are similar to the arrangement highlighted in Figure $11 \mathrm{~B}$. We address this issue in more detail below.

Additional architectural features of the directional preference maps are highlighted in Figures 13 and 14. A miniaturized reproduction of Figure $12 \mathrm{~A}$ appears in Figure $13 A$ for spatial reference. The highlighted region in Figure $13 B$ contains a pair of directional singularities (at center and bottom right) linked by a directional fracture $\left(180^{\circ}\right.$ reversal). Each singularity forms the center of a half-rotation $\left(180^{\circ}\right)$ pinwheel with fracture. Interestingly, these paired pinwheels are of opposite rotational sign (i.e., clockwise and counterclockwise). Also illustrated are the neuronal responses recorded at each of the five indicated map locations in Figure $13 B$. Responses at the recording site located close to the central pinwheel singularity reveal weak bidirectionality that was shaped by inhibition in a manner consistent with the trend noted above. Neuronal responses at the other four recording sites were primarily excitatory and unidirectional. (The leftmost site was only weakly responsive, with a directional preference determined by opposing excitatory and inhibitory influences.)

Figure 14 highlights two regions extracted from the directional map in Figure $12 \mathrm{~A}$, in which preferred directionchanged smoothly and continuously. Once again, a miniaturized reproduction of Figure $12 A$ appears in Figure $14 A$ for spatial reference. Figure 14, $B$ and $C$, illustrates the relevant map regions along with neuronal responses from the indicated recording sites. The illustrated responses, which were recorded far from any discontinuities, are unidirectional and highly selective. In both highlighted cases, the neuronal responses reveal gradual progressions of preferred direction, which are reflected in the colorcoded directional maps.

\section{Evaluation of interpolation precision}

The range of estimated error in the interpolation of preferred direction of motion was computed as a function of displacement between measured and interpolated map sites (see Materials and Methods). These error values are plotted in Figure 15 for displacements within the sampling regions $(350 \times 200 \mu \mathrm{m})$ bounded by four recording sites. The height of each grid vertex represents the SD of the error distribu- 
tion for the indicated map displacement at all equivalent position in the maps shown in Figures $11 A$ and $12 A$. The interpolation error was, of course, zero at the recording sites. In contrast, the interpolation error was estimated to be $37^{\circ}$ at the maximum displacement from the recording sites. The average interpolation error was $24^{\circ}$. Both values are significantly smaller than the average directional tuning bandwidth for gratings $\left(105^{\circ}\right)$ or for dots $\left(125^{\circ}\right)$, which demonstrates that we have been able to interpolate our $2 \mathrm{D}$ directional-preference maps with a meaningful degree of precision.

\section{Module size and periodicity in \\ directional maps}

The directional parameter that we have studied is inherently periodic. To better appreciate the functional significance of the observed cortical maps, we attempted to evaluate the periodicity and scale of the directional representation in the cortex. As shown above, the $2 \mathrm{D}$ composite maps are characterized by slow continuous changes in preferred direction. These progressions are interrupted, however, by discontinuities that fragment the directional representation into patches. In addition, radial arrangements are embedded in continuous bands of direction selectivity. Although such mosaics lack the regularity needed to derive precise measurements of periodicity, other investigators have approached this issue using spatialfrequency decomposition methods (2D Fourier analysis) or gradient functions to derive the rate of change of the mapped stimulus parameter. Each method has advantages and disadvantages, and we have thus applied a combination in an effort to identify consistent patterns.

Our single-condition maps individually represent firing rates to a single direction of motion. Because the representation of direction is continuous, periodicities in the patterns of responses seen in these maps should reflect the period over which a cycle $\left(360^{\circ}\right)$ of stimulus direction (i.e., a functional module) is represented. We applied 2D Fourier analysis to all of our single-condition maps. The resulting spatial-frequency power spectra revealed a consistent and significant energy peak in the majority of maps, which was centered on $1.60-1.25$ cycles $/ \mathrm{mm}$. This corresponds to a period of $600-800$ $\mu \mathrm{m}$ for a complete cycle of stimulus direction $\left(360^{\circ}\right)$. We also evaluated directional modules in the single-condition maps using a nearest-neighbor analysis of firingrate peaks (effectively a spatial-domain
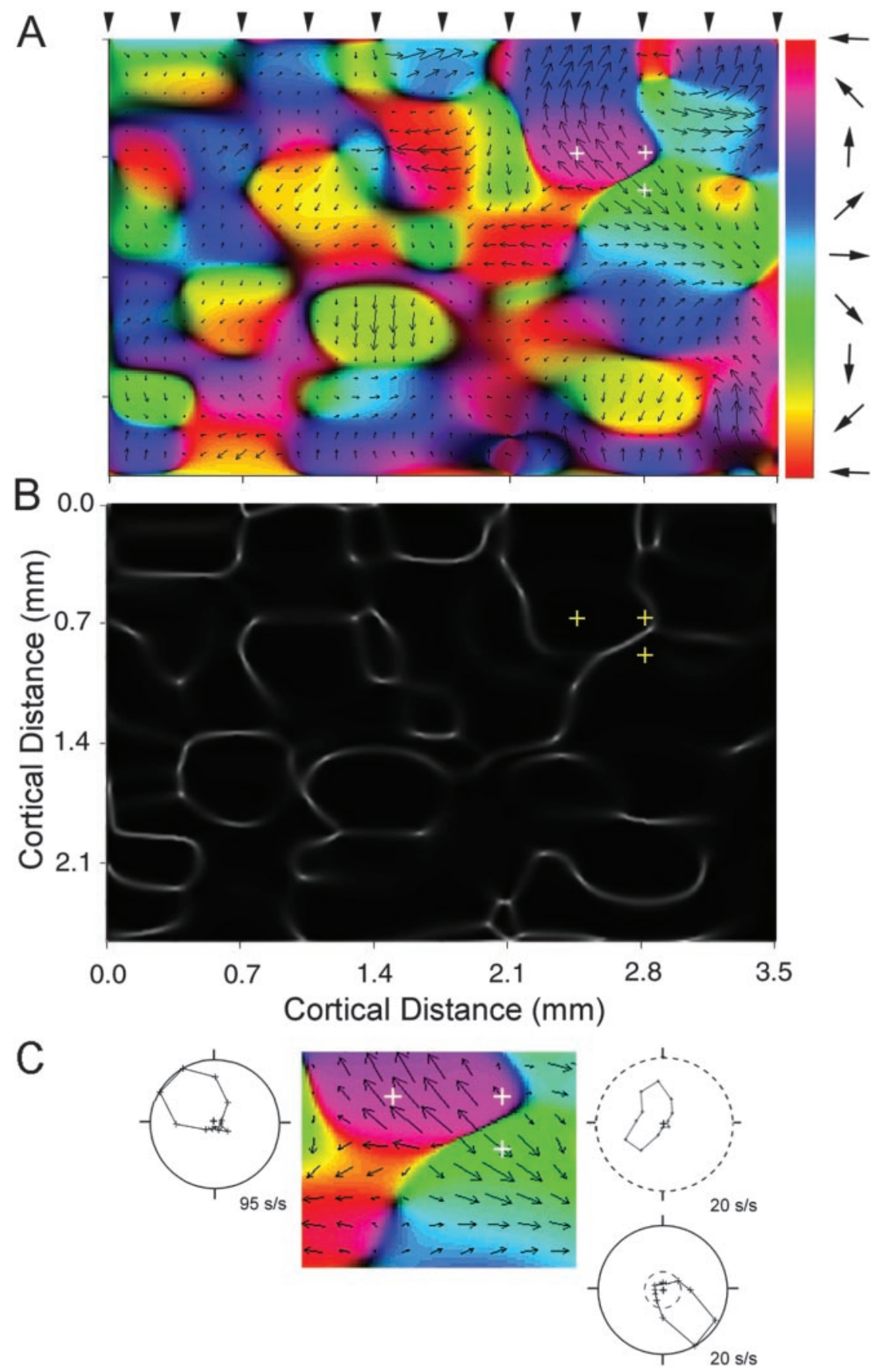

Figure 11. A, Color-coded composite map of the two-dimensional surface of area MT, which represents the preferred direction for a moving grating. These maps were computed by multiplying each of the 12 single-condition neuronal response maps shown in Figure 10 by a vector corresponding to the direction of stimulus motion and then taking the sum of the resulting vector matrices (see Materials and Methods). Small arrowheads at top indicate trajectories of the 11 electrodes in the array. Color code for preferred direction of motion appears along the right margin of the map. Arrows overlaid on the color map are a subsampled vector description of local directional preference; arrow direction represents the preferred direction (redundant with color code), and relative length reflects the strength of selectivity. Dark regions of the color-coded map indicate areas for which measurements were deemed unreliable, as calculated by a bootstrap algorithm. $B$, Directional preference discontinuities (fractures and singularities) in the composite map of $A$ can be identified in the $2 \mathrm{D}$ gradient map, which illustrates the rate of change of preferred direction. The map was computed using the gradient transform (bright denotes high rate of change). Thus, the bright areas correspond to regions at which preferred direction underwent a sharp transition. $C$, Magnified view of a directional discontinuity (fracture) present in the top-right region of the map shown in $A$. This fracture extends from bottom left to top right and is most evident from the red/green transition. The three crosshairs (also shown in $A$ and $B$ ) indicate the locations of three recording sites that span the fracture. Directional tuning curves obtained at these threesites areillustrated in polar format. Indicated spike rates correspond to the scale of the outer circle in each plot. Broken circles indicate spontaneous activity level. The tuning curve at top left corresponds to the recording site highlighted in Figure 10. The tuning curve at bottom right shows a recording on the opposite side of the fracture, which was selective for the opposite direction of motion. The remaining recording site (top right) is located very near the fracture, and the tuning curve shows that its directional preference was primarily shaped by inhibition. 


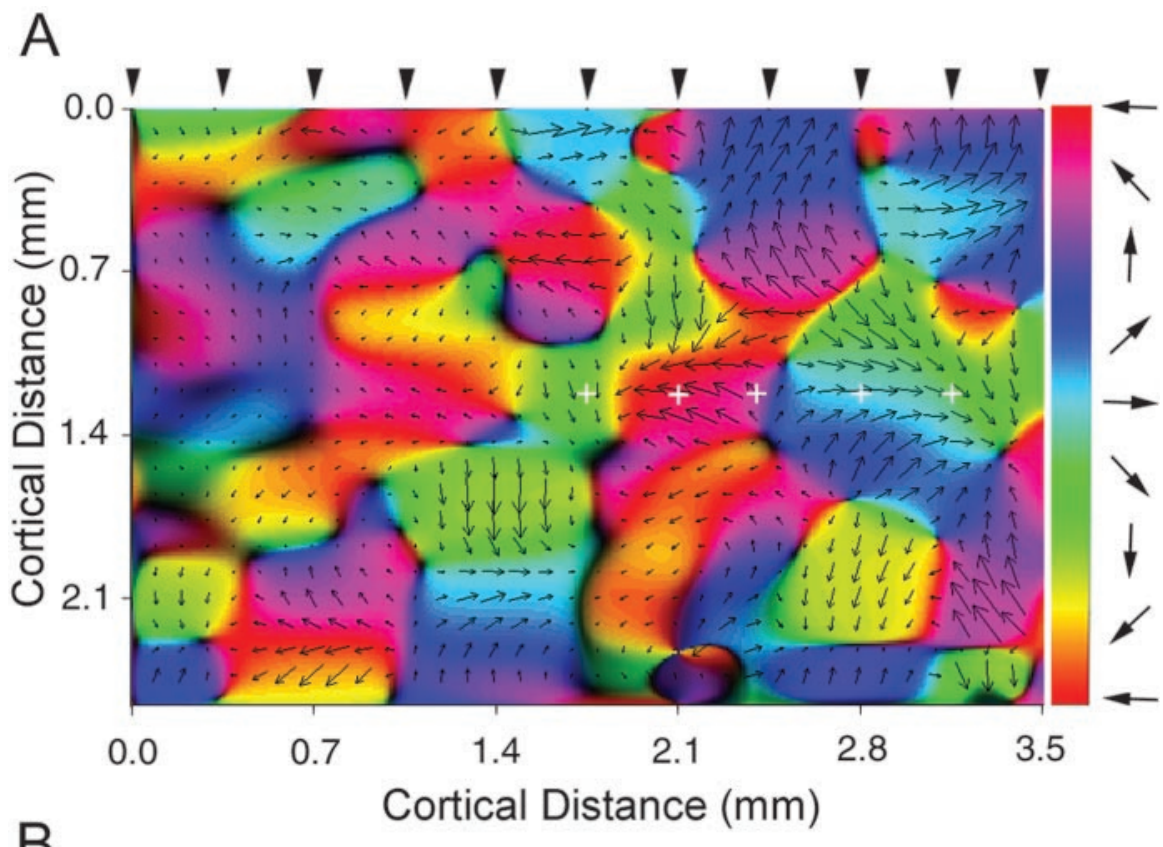

B
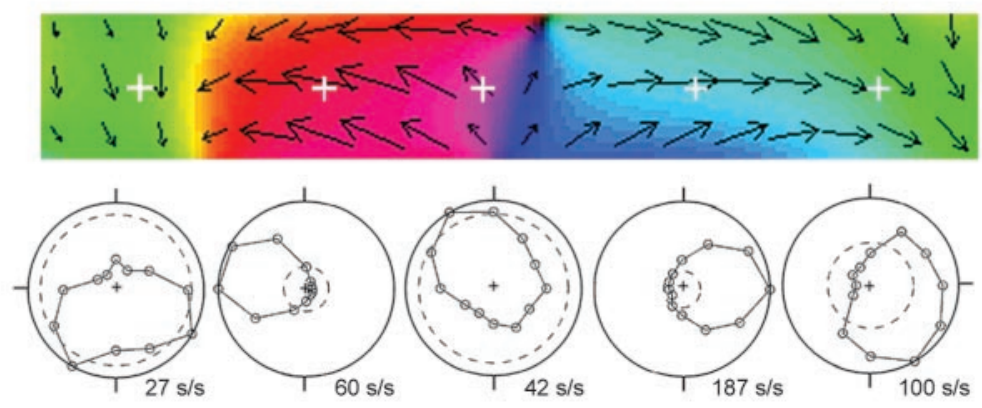

Figure 12. $\quad A$, Color-coded composite map of the two-dimensional surface of area MT, which represents the preferred direction for a moving dot stimulus. This map is coextensive with that of Figure $11 \mathrm{~A}$ and is derived from the same recording sites. In comparison with Figure $11 \mathrm{~A}$, this map serves to illustrate the marked similarities between functional maps generated using moving dots versus moving gratings, which suggest that the directional preferences of area MT neurons in Cebus are not substantially sensitive to the form of the moving stimulus. Map derivation and plotting conventions are the same as in Figure $11 A$. $B$, Magnified view of a strip of cortex drawn from the right-center portion of the map shown in $A$, in which the rate of change of preferred direction varied considerably. Much of this strip consists of gradual shifts in preferred direction, which were interrupted by two directional fractures (identifiable by the thin yellow and purple bands). The five white crosshairs indicate the locations of recording sites that are within regular sequences and span fractures. Directional tuning curves obtained at these five sites are illustrated at the bottom. Tuning curves are illustrated in polar format. Spike rates $(\mathrm{s} / \mathrm{s})$ correspond to the scale of the outer circle in each plot. Broken circles indicate spontaneous activity level. The two sites nearest the two fractures (first and third from left) exhibited directional tuning that was shaped primarily by inhibition. In contrast, sites in the midst of smooth sequences exhibited highly excitatory responses and were unidirectional. s/s, Spikes per second.

analysis of periodicity in thresholded maps). This method yielded a distribution of interpeak distances with a median of $613 \mu \mathrm{m}$ (first quartile, $545 \mu \mathrm{m}$; third quartile, $783 \mu \mathrm{m}$ ), which supports the estimate of module size obtained using spatial-frequency analysis.

Module size measurements can also be derived by assessing the average rate of change of preferred direction from the composite maps. To obtain these measurements, we first applied a 2D gradient operator. Because the maps were fragmented by discontinuities (at which rate of change could approach infinity), we thresholded gradients at a value equal to twice the mean. The resulting distribution was used to compute an average rate of smooth changes (i.e., without discontinuities) of preferred direction of motion. The value obtained for the map illustrated in Figure 11 was $0.36^{\circ} / \mu \mathrm{m}$; similar values were obtained for other maps. This gradient measure argues that a complete linear directional cycle can be represented, on average, in $1 \mathrm{~mm}$ of cortex.

Estimates of module periodicity and size yielded by the spatial-frequency and interpeak-distance analyses were thus somewhat smaller $(\sim 20-40 \%)$ than that obtained by the gradient analysis. This is not terribly surprising given the fractured nature of the directional maps. Indeed, the spatial-frequency and distance measures, which were obtained from singlecondition response maps, are likely to be skewed simply because they do not take map discontinuities into account. We thus consider the smallest period $\left(\sim 360^{\circ} \%\right.$ $600 \mu \mathrm{m}$ ) obtained by these analyses to be a lower bound. Although gradient measures may be susceptible to thresholding biases, we believe that the module size obtained by these means $\left(360^{\circ} / 1 \mathrm{~mm}\right)$ is a more conservative estimate. We address the significance of this value in Discussion.

Inhibitory versus excitatory influences on neuronal responses

As noted above, comparisons between the 2D directional preference maps and the measured neuronal responses at sites within those maps indicated that map discontinuities were commonly associated with selectivity patterns that were primarily shaped by inhibition. To evaluate this suggestion more systematically, we computed an index of inhibition that quantifies the relative magnitude of inhibitory and excitatory contributions to the directional tuning at each recording site (see Materials and Methods). We examined this index as a function of distance from each directional discontinuity in the corresponding 2D map of directional preferences. Not surprisingly, the excitatory contribution to directional tuning was stronger than the inhibitory contribution for the majority of recording sites. Sites exhibiting a relatively large degree of excitatory contribution were located everywhere in the map, including very close to discontinuities. In contrast, the frequency of sites exhibiting a relatively large degree of inhibition was greater for sites located close to a discontinuity (up to $\sim 150 \mu \mathrm{m}$ ) than for more distant sites. Indeed, the index of inhibition declined significantly with distance from the nearest discontinuity (angular coefficient $=-0.001 ; p<0.042)$.

\section{Discussion}

We have used a novel technique, which we term electrophysiological imaging, to establish the 2D layout of functional maps in cortical visual area MT of the New World Cebus monkey. This method has a distinct advantage over the optical imaging approach: it is applicable in optically inaccessible regions of the 

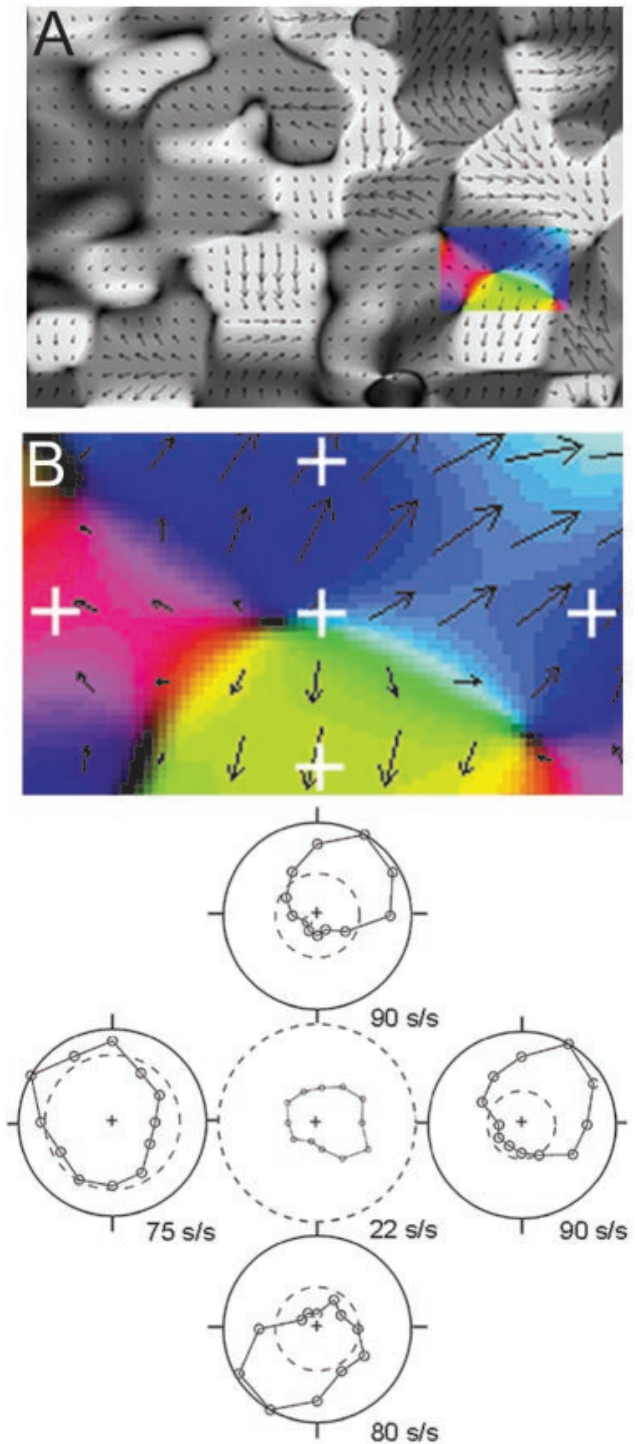

Figure 13. Illustration of neuronal responses that gave rise to a pinwheel map formation. $A$, Miniaturized reproduction of preferred direction map (dot stimulus) from Figure 12A, which illustrates the location of the map region highlighted in $B$. $B$, Magnified view of a rectangular region of cortex extracted from map in $A$, which illustrates a pair of directional singularities (at center and bottom right) with corresponding pinwheel formations, which are linked by a fracture. Each pinwheel is composed of a half-rotation $\left(180^{\circ}\right)$ and a fracture. White crosshairs indicate the locations of five recording sites, which include a site near the pinwheel center and four sites around the perimeter. Directional tuning curves obtained at these sites are illustrated at bottom in polar format. Spike rates $(\mathrm{s} / \mathrm{s})$ correspond to the scale of the outer circle in each plot. Broken circles indicate spontaneous activity level. The central site exhibited a weak form of directional tuning that was shaped entirely by inhibition. The remaining sites were excitatory and unidirectional.

brain such as area MT. Our findings document the presence in area MT of directional map features that have been discovered by OI in other cortical areas (Blasdel and Salama, 1986; Ts'o et al., 1990; Bonhoeffer and Grinvald, 1991; Malonek et al., 1994; Weliky et al., 1996). In the remainder of Discussion, we address specific issues raised by our results and their relationship to previous findings.

\section{Electrophysiological imaging versus optical imaging}

The use of multielectrode arrays, in combination with firing-rate interpolation and vector sum algorithms borrowed from OI,

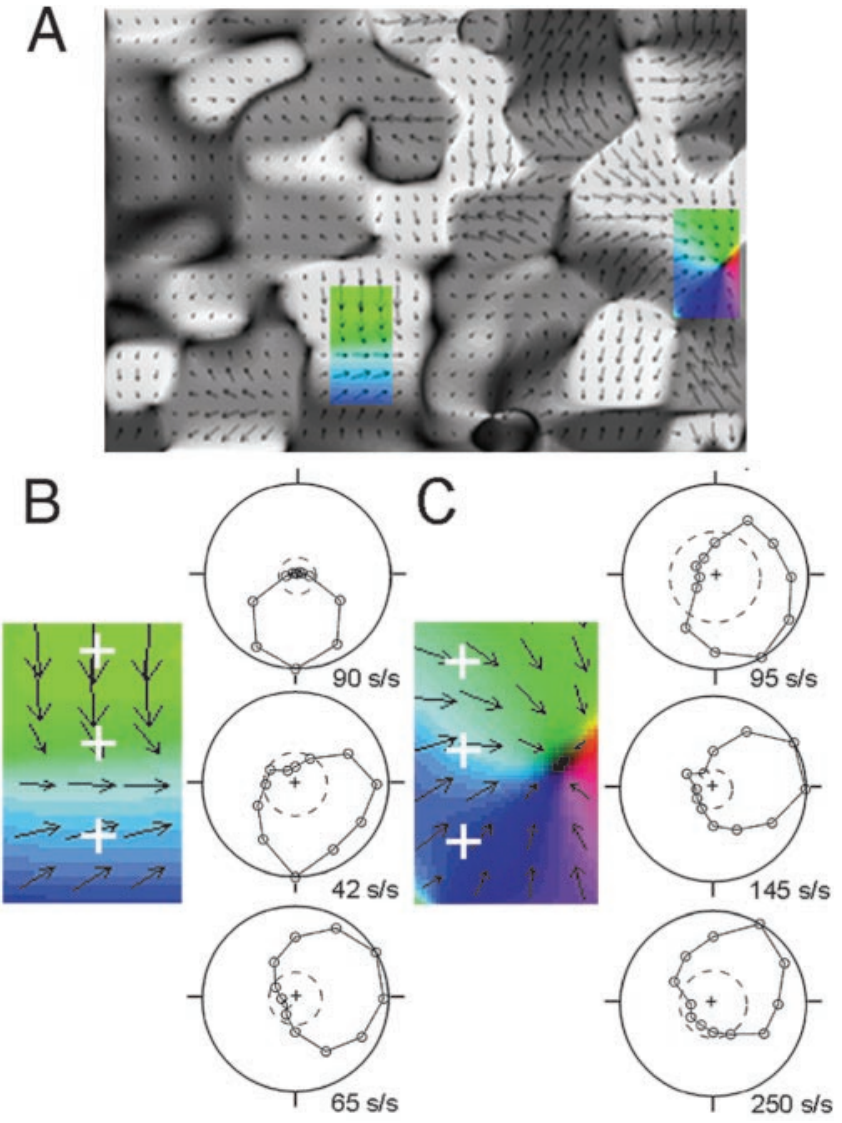

Figure 14. Illustration of neuronal responses associated with linear and radial progressions of preferred direction of motion. $A$, Miniaturized reproduction of preferred direction map from Figure $12 A$, which illustrates the locations of map regions highlighted in $B$ and C. $B$, Magnified view of a rectangular region of cortex extracted from lower central region of map in $A$, which illustrates a smooth linear progression of preferred direction of motion. White crosshairs indicate the locations of three recording sites along this progression. Directional tuning curves obtained at these three sites are illustrated at right in polar format. Spike rates $(\mathrm{s} / \mathrm{s})$ correspond to the scale of the outer circle in each plot. Broken circles indicate spontaneous activity level. All three recording sites exhibited strong unidirectional responses. C, Magnified view of a rectangular region of cortex extracted from rightward region of map in $A$, which illustrates another smooth progression of preferred direction of motion. White crosshairs indicate the locations of three recording sites along different radial axes. Directional tuning curves obtained at these three sites are illustrated at right in polar format. All three recording sites exhibited strong unidirectional responses.

have enabled us to develop EI as a complementary alternative. Its primary advantage is its applicability under conditions in which OI is impossible because of the absence of an optical path. Additional advantages include the potential for a layer-specific analysis, knowledge of the patterns of neuronal tuning that give rise to map features, and superior temporal resolution (Fig. 1). EI is also preferable to OI for many applications because the optical response is only indirectly related to the activity of individual neurons and may be influenced by local synaptic currents, subthreshold membrane depolarizations, and hemodynamic components unrelated to neuronal spiking, as well as by out-of-focus signals from different layers. Electrophysiological recording clearly does not have these disadvantages.

Nonetheless, there are some weaknesses to the electrophysiological approach. First, in the form applied here, the spatial resolution is not as great as that of typical OI applications. Although there may be room for improvement, the resolution of EI may take some time to approach that of OI. Second, because EI (as 
$0^{\circ} \quad$ Error (deg)
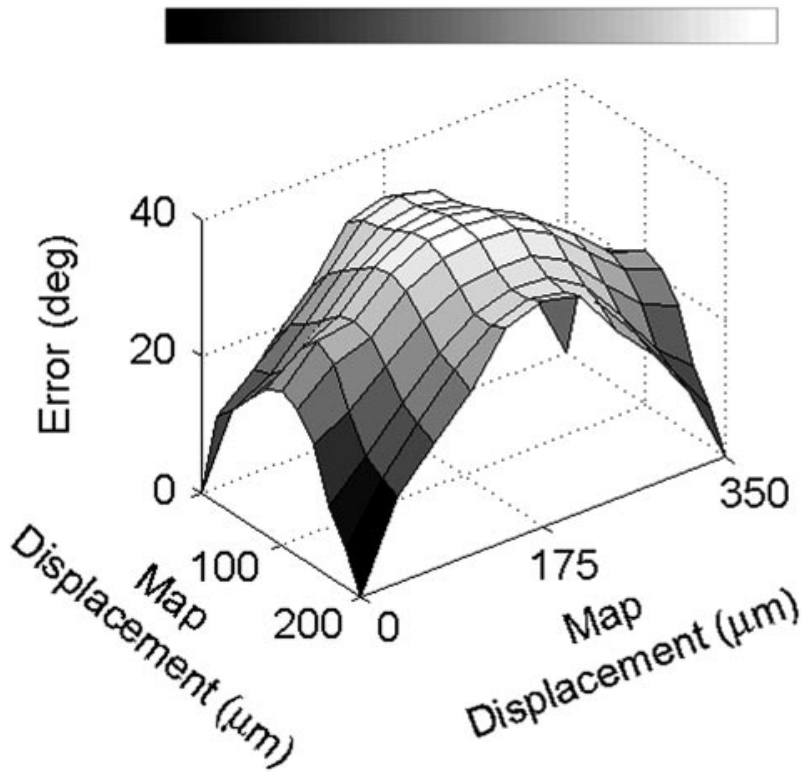

Figure 15. Estimated error in the interpolation of preferred direction of motion as a function of $2 \mathrm{D}$ map position within the sampling region bounded by measured map values. Each small square $(35 \times 20 \mu \mathrm{m})$ represents the SD of the error distribution at all equivalent positions in the directional maps shown in Figures $11 \mathrm{~A}$ and $12 \mathrm{~A}$. The interpolation error was naturally zero at the recording sites. The error estimate reached its largest value $\left(37^{\circ}\right)$ at the maximum distance from the recording sites. The average interpolation error was $24^{\circ}$. Gray-scale values are proportional to interpolation error; lighter areas represent larger errors. See Materials and Methods for the interpolation error analysis procedure.

used in our experiments) requires that different parts of the map be sampled at different times, we must assume that neuronal tuning does not vary over the time required to obtain samples. This assumption may not hold under conditions in which behavior is varying over time. New 2D multielectrode arrays may overcome this limitation. Third, EI is more invasive than OI. Our data demonstrate that any tissue damage caused by the electrode array was not so great as to disrupt neuronal tuning (Figs. $6 D, 7 D$ ) but damage might be manifested as disruption of RF surround properties, which were not examined systematically in the present study. OI avoids these problems, although the massive retraction of dura required for that approach introduces a different set of concerns [but see Shtoyerman et al. (2000)].

\section{Functional map features revealed by electrophysiological imaging}

General features of the EI maps presented herein bear a striking resemblance to OI data, suggesting that they may reflect similar forms of neuronal circuitry and can be interpreted in a similar manner. For example, we have observed pinwheel-like modules for directional preference, similar to those identified for orientation and direction in area 18 of cat visual cortex (Bonhoeffer and Grinvald, 1991; Shmuel and Grinvald, 1996) and area 17 of ferret (Weliky et al., 1996) and macaque (Blasdel and Salama, 1986; Ts'o et al., 1990), as well as area MT of owl monkey (Malonek et al., 1994). We also observed bands in which directional preference changed gradually across the cortical surface of MT, as described previously by Albright et al. (1984) in the macaque. These regular sequences were often interrupted by reversals of preferred direction of motion. A similar pattern of reversals was also seen in the macaque and was incorporated in a $2 \mathrm{D}$ functional model that predicted the existence of directional fractures (Albright et al., 1984). Subsequent studies of the $2 \mathrm{D}$ organization of directional preferences in cat area 18 (Swindale et al., 1987; Bonhoeffer and Grinvald, 1991; Shmuel and Grinvald, 1996), ferret area 17 (Weliky et al., 1996), and owl monkey area MT (Malonek et al., 1994) obtained direct evidence for the predicted fractures, as well as directional singularities centered on pinwheels. The present study confirms the existence of such directional discontinuities in Cebus area MT.

\section{Functional architecture, local circuitry, and inhibitory influences on directional tuning}

\section{Inhibition and map discontinuities}

Properties of the recording sites highlighted in Figures 11-13 suggest a spatial relationship between map discontinuities and inhibitory influences on neuronal tuning. Our findings complement those of Roerig and Kao (1999), who investigated the relationship between intrinsic cortical connections and the spatial representation of directional preference in area 17 of ferret. These investigators found that excitatory and inhibitory synaptic inputs to directionally selective neurons were isodirection tuned. However, $40 \%$ of the inhibitory connections originated in regions preferring the direction opposite from that represented at their point of termination. These findings, in conjunction with our own data, suggest that some directional fractures are spanned by circuits of reciprocal inhibition, such that the preferred direction on one side of a fracture is the direction eliciting the strongest inhibition on the other side and vice versa. Intermediate map locations (i.e., those very near a fracture) may receive inhibitory contributions from both sides. The patterns of neuronal selectivity highlighted in Figures $11 B, 12 B$, and $13 B$, in which responses near directional discontinuities appear to be shaped by inhibition, may be manifestations of this hypothetical circuit.

\section{Are singularities unique?}

Maldonado et al. (1997) reported that orientation pinwheel centers in cat areas 17 and 18, which lack a distinct orientation preference in optical maps (by definition), are populated by neurons that are highly selective for stimulus orientation. The lack of selectivity in the optical map may be accounted for by high variability among the orientation preferences of neighboring cells. In contrast, map regions of high orientation selectivity are populated by selective neurons with similar preferences. Because the properties of individual neurons in pinwheel centers are thus indistinguishable from those of iso-orientation domains, Maldonado et al. (1997) concluded that pinwheel centers do not constitute distinct functional compartments. These findings and the conclusion drawn appear to conflict with our claim that the incidence of recording sites exhibiting a relatively large degree of inhibition was greatest for sites located close to a discontinuity (e.g., pinwheel centers and fractures). There are a number of important methodological differences between our study and that of Maldonado et al. (1997), which may account for the discrepancy. For example, Maldonado et al. (1997) investigated single neurons in area V1 of cats anesthetized with halothane, and we studied multiunit activity in area MT of monkeys anesthetized with fentanyl citrate and $\mathrm{N}_{2} \mathrm{O} / \mathrm{O}_{2}$. Additional experiments are needed to better understand these and other factors contributing to the similarities and discrepancies between the results reported. 


\section{Functional modularity: relationship between directionality and visual field topography}

The coexistence of a spatially repeating representation of direction and a topographic representation of visual space in Cebus area MT potentially allows for a full complement of directional detectors to be present at each location in the visual field map. In particular, the size of the cortical representation of each neuronally resolvable visual field coordinate (the point image size) should equal or exceed the size of a complete representation of the relevant stimulus parameter. Hubel and Wiesel (1974b) obtained evidence in support of this hypothesis for orientation in macaque V1. Similarly, Albright and Desimone (1987) found that the representation of direction $\left(360^{\circ} / 860 \mu \mathrm{m}\right)$ was well within the point image size throughout macaque MT. The average rate of change of preferred direction in Cebus area MT $\left(360^{\circ} / 1\right.$ $\mathrm{mm}$ ) is comparable with that of macaques. In light of known similarities between the visual field representations of Cebus and macaque area MT (Fiorani et al., 1989), our data suggest that Cebus also possess a full complement of motion detectors for each location in the visual field map.

\section{Homologies between New World and Old World monkeys}

The New World (Platyrrhine) monkey Cebus apella has been the subject of a number of recent studies aimed at elucidating visual system organization and function (Gattass et al., 1987; Rosa et al., 1988, 1993; Fiorani et al., 1989; Sousa et al., 1991). Despite a distant point of evolutionary divergence, these studies have revealed some extraordinary parallels between the Cebus monkey and the Old World (Catarrhine) macaque, which is the most widely studied nonhuman primate model for vision. There are strong similarities between the brain sizes and cortical sulcal patterns of these two species, and they occupy similar behavioral niches (Fleagle, 1988). There also exists an abundance of physiological and anatomical evidence for homologies of cortical visual areas between the two species and a striking correspondence with regard to the positions of homologous areas relative to gyral morphology. The present demonstration of a similar cortical architecture for directionality in macaque and Cebus area MT adds additional evidence for homology and highlights the benefit of comparative studies for identifying general principles of visual cortical organization and function.

\section{References}

Albright TD (1984) Direction and orientation selectivity of neurons in visual area MT of the macaque. J Neurophysiol 52:1106-1130.

Albright TD (1989) Centrifugal directional bias in the middle temporal visual area (MT) of the macaque. Vis Neurosci 2:177-188.

Albright TD, Desimone R (1987) Local precision of visuotopic organization in the middle temporal area (MT) of the macaque. Exp Brain Res 65:582-592.

Albright TD, Desimone R, Gross CG (1984) Columnar organization of directionally selective cells in visual area MT of the macaque. J Neurophysiol 51:16-31.

Batschelet E (1981) Circular statistics in biology. London: Academic.

Blasdel GG (1992) Orientation selectivity, preference, and continuity in monkey striate cortex. J Neurosci 12:3139-3161.

Blasdel GG, Salama G (1986) Voltage-sensitive dyes reveal a modular organization in monkey striate cortex. Nature 321:579-585.
Bonhoeffer T, Grinvald A (1991) Iso-orientation domains in cat visual cortex are arranged in pinwheel-like patterns. Nature 353:429-431.

Braitenberg V, Braitenberg C (1979) Geometry of orientation columns in the visual cortex. Biol Cybern 33:179-186.

Fiorani Jr M, Gattass R, Rosa MG, Sousa AP (1989) Visual area MT in the Cebus monkey: location, visuotopic organization, and variability. J Comp Neurol 287:98-118.

Fleagle JG (1988) Primate adaptation and evolution. San Diego: Academic.

Gallyas F (1979) Silver staining of myelin by means of physical development. Neurol Res 1:203-209.

Gattass R, Gross CG (1981) Visual topography of striate projection zone (MT) in posterior superior temporal sulcus of the macaque. J Neurophysiol 46:621-638.

Gattass R, Sousa AP, Rosa MG (1987) Visual topography of V1 in the Cebus monkey. J Comp Neurol 259:529-548.

Grinvald A, Shoham D, Shmue A, Glaser D, Vanzetta I, Shtoyermann E, Slovin H, Sterkin A, Wijnbergen C, Hildesheim R, Arieli A (2001) In vivo optical imaging of cortical architecture and dynamics. In: Modern techniques in neuroscience research, 893-969. New York: Springer.

Hubel DH, Wiesel TN (1962) Receptive fields, binocular interaction and functional architecture in the cat's visual cortex. J Physiol (Lond) 160:106-154.

Hubel DH, Wiesel TN (1974a) Sequence regularity and geometry of orientation columns in the monkey striate cortex. J Comp Neurol 158:267-294.

Hubel DH, Wiesel TN (1974b) Uniformity of monkey striate cortex: a parallel relationship between field size, scatter, and magnification factor. J Comp Neurol 158:295-305.

Maldonado PE, Godecke I, Gray CM, Bonhoeffer T (1997) Orientation selectivity in pinwheel centers in cat striate cortex. Science 276:1551-1555.

Malonek D, Tootell RB, Grinvald A (1994) Optical imaging reveals the functional architecture of neurons processing shape and motion in owl monkey area MT. Proc R Soc Lond B Biol Sci 258:109-119.

Mountcastle VB (1957) Modality and topographic properties of single neurons of cat's somatic sensory cortex. J Neurophysiol 20:408-434.

Roerig B, Kao JPY (1999) Organization of intracortical circuits in relation to direction preference maps in ferret visual cortex. J Neurosci 19:1-5.

Rosa MG, Sousa AP, Gattass R (1988) Representation of the visual field in the second visual area in the Cebus monkey. J Comp Neurol 275:326-345.

Rosa MG, Soares JG, Fiorani Jr M, Gattass R (1993) Cortical afferents of visual area MT in the Cebus monkey: possible homologies between New and Old World monkeys. Vis Neurosci 10:827-855.

Shmuel A, Grinvald A (1996) Functional organization for direction of motion and its relationship to orientation maps in cat area 18. J Neurosci 16:6945-6964.

Shtoyerman E, Arieli A, Slovin H, Vanzetta I, Grinvald A (2000) Long-term optical imaging and spectroscopy reveal mechanisms underlying the intrinsic signal and stability of cortical maps in V1 of behaving monkeys. J Neurosci 20:8111-8121.

Sousa AP, Pinon MC, Gattass R, Rosa MG (1991) Topographic organization of cortical input to striate cortex in the Cebus monkey: a fluorescent tracer study. J Comp Neurol 308:665-682.

Swindale NV, Matsubara JA, Cynader MS (1987) Surface organization of orientation and direction selectivity in cat area 18. J Neurosci 7:1414-1427.

Ts'o DY, Frostig RD, Lieke EE, Grinvald A (1990) Functional organization of primate visual cortex revealed by high resolution optical imaging. Science 249:417-420.

Ungerleider LG, Mishkin M (1979) The striate projection zone in the superior temporal sulcus of Macaca mulatta: location and topographic organization. J Comp Neurol 188:347-366.

Van Essen DC, Maunsell JHR, Bixby JL (1981) The middle temporal visual area in the macaque: myeloarchitecture, connections, functional properties and topographic connections. J Comp Neurol 199:293-326.

Weliky M, Bosking WH, Fitzpatrick D (1996) A systematic map of direction preference in primary visual cortex. Nature 379:725-728. 